\title{
Full and One Dimension
}

Samuel M. Greene, ${ }^{1, \text { a) }}$ Xiao Shan, ${ }^{1}$ and David C. Clary $^{1}$

Physical and Theoretical Chemistry Laboratory, Department of Chemistry, University of Oxford, South Parks Road, Oxford, OX1 3QZ,

United Kingdom

(Dated: 29 April 2016)

\section{ABSTRACT}

Semiclassical Transition State Theory (SCTST), a method for calculating rate constants of chemical reactions, offers gains in computational efficiency relative to more accurate quantum scattering methods, In full-dimensional (FD) SCTST, reaction probabilities are calculated from third and fourth potential derivatives along all vibrational degrees of freedom. However, the computational cost of FD SCTST scales unfavorably with system size, which prohibits its application to larger systems. In this study, the accuracy and efficiency of 1-D SCTST, in which only third and fourth derivatives along the reaction mode are used, are investigated in comparison to those of FD SCTST. Potential derivatives are obtained from numerical ab initio Hessian matrix calculations at the MP2/cc-pVTZ level of theory, and Richardson extrapolation is applied to improve the accuracy of these derivatives. Reaction barriers are calculated at the CCSD $(\mathrm{T}) / \mathrm{cc}-\mathrm{pVTZ}$ level. Results from FD SCTST agree with results from previous theoretical and experimental studies when Richardson extrapolation is applied. Results from our implementation of 1-D SCTST, which uses only 4 single-point MP2/cc-pVTZ energy calculations in addition to those for conventional TST, agree with FD results to within a factor of 5 at $250 \mathrm{~K}$. This degree of agreement and the efficiency of the 1-D method suggest its potential as a means of approximating rate constants for systems too large for existing quantum scattering methods.

a)Electronic mail: samuel.greene@chem.ox.ac.uk 


\section{Publishi.hg INTRODUCTION}

Theoretical methods for calculating rate constants of chemical reactions have useful applications in a variety of areas, including atmospheric chemistry, ${ }^{1-4}$ biological chemistry, ${ }^{5}$ and materials science. ${ }^{6-8}$ Quantum reactive scattering (QRS) approaches involve solving the nuclear Schrödinger equation on a potential energy surface (PES) and often yield the most accurate rate constants. ${ }^{9-15}$ However, constructing a suitably accurate PES and performing a full-dimensional (FD) calculation become very computationally demanding as the size of the system increases. ${ }^{16-19}$ To our knowledge, despite recent advances in both of these areas, ${ }^{20-30}$ the applicability of FD QRS methods remains limited to reactions involving six atoms. ${ }^{29,31-40}$

This limitation has prompted the development of a variety of approximate methods for dynamics calculations. Variational Transition State Theory with Multidimensional Tunneling corrections (VTST/MT) constitutes a class of methods in which reaction probabilities are calculated semiclassically from action integrals along paths on the PES. ${ }^{41-43}$ It is often possible to obtain results in good agreement with benchmark QRS results with an appropriate choice of a VTST/MT method. ${ }^{4-52}$ Other methods that require a complete PES include include quantum mechanical formulations of Transition State Theory ${ }^{53-56}$ and the ring polymer molecular dynamics (RPMD) method. ${ }^{57,58}$ Both of these methods have yielded accurate results for a variety of reactions. ${ }^{44,45,48,59,60}$

Semiclassical Transition State Theory (SCTST) is another approximate theory and was developed originally by Miller et al. ${ }^{61-63}$ It differs from the aforementioned theories in that it depends on the PES and its derivatives only at the transition state (TS). In SCTST, reaction probábilities are calculated semiclassically from an expression for the energy at the TS in terms of the system's "good" quantum numbers. This expression is commonly derived using second-order vibrational perturbation theory (VPT2), which depends only upon second, third, and fourth derivatives of the PES (i.e., a quartic force field). Evaluating these derivatives is often more efficient than constructing a complete PES. This efficiency gain has been a primary motivation in the development and application of SCTST, which has been applied previously to reactions such as $\mathrm{H}+\mathrm{H}_{2} \longrightarrow \mathrm{H}_{2}+\mathrm{H},{ }^{64} \mathrm{HBr}+\mathrm{Cl} \longrightarrow$ $\mathrm{Br}+\mathrm{HCl},{ }^{65} \mathrm{HO}+\mathrm{H}_{2} \longrightarrow \mathrm{H}_{2} \mathrm{O}+\mathrm{H}$ and isotopologues, ${ }^{66}$ and $\mathrm{Cl}+\mathrm{CH}_{4} \longrightarrow \mathrm{HCl}+\mathrm{CH}_{3}$ and isotopologues. ${ }^{67}$ In all of these cases, results from SCTST have exhibited good agreement 
Publishiwgig those from other theories and from experiment.

Previously, ${ }^{68}$ we evaluated a reduced-dimensionality (RD) approach to SCTST, in which only a subset of the system's degrees of freedom (DOFs) are included explicitly in calculations of the reaction probability (i.e., via calculation of a quartic force field) while the remaining DOFs are treated harmonically with a partition function. In an earlier study, Nguyen et al. ${ }^{66}$ implemented a one-dimensional (1-D) approach to SCTST. We previously applied this approach to four reactions ${ }^{69}$ and found that the deep tunneling corrections to this theory described by Wagner ${ }^{70}$ had to be applied to ensure close agreement with results from accurate QRS calculations performed on the same 2-D PESs. It should be noted that 1-D SCTST with deep tunneling corrections is similar to the asymmetrical Eckart method implemented in Ref. 66 but differs in that (1) it treats the anharmonicity of the reaction mode and (2) it can be improved systematically by treating more DOFs.

These previous findings suggest that RD SCTST calculations can yield suitable estimates of rate constants from higher-dimensionality calculations. Typical implementations of full-dimensional (FD) SCTST calculations require on the order of $F$ ab initio frequency calculations, where $F$ is the number of internal DOFs of the system. ${ }^{66,67}$ The computational cost of RD SCTST calculations scales more favorably than that of FD calculations with respect to system size, which suggests the applicability of the RD SCTST method to systems larger than those that ean be treated using other theories. The primary objective of this study is to evaluate whether good estimates of rate constants can be obtained using 1-D SCTST, as judged by comparison with results from FD SCTST, and to compare the computational costs of these methods. More rigorous treatments that involve greater computational expense are possible for the systems considered in this study, but the aim here is to develop a practical method that can be applied to larger systems.

The quartic force field required for FD SCTST calculations is often obtained by differentiating the Hessian matrix of second derivatives of the PES numerically. ${ }^{71-73}$ This approach has been applied previously in cases in which analytical first or second derivatives are available. ${ }^{66,67,71-76}$ Many electronic structure software packages enable the efficient calculation of analytical first and second derivatives at various levels of theory, although analytical derivatives for open-shell systems are not as widely implemented as for closedshell systems. ${ }^{77-81}$ Many of the elementary gas-phase chemical reactions studied previously have open-shell transition state structures, ${ }^{15,31,32,40,82,83}$ including those reactions considered 
We discuss an approach to quartic force field calculations that does not require analytical derivatives. An advantage of this approach is that it is available to users of all electronic structure packages at all levels of theory. In some cases, calculations of analytical second derivatives are computationally more expensive than numerical calculations, so this approach may offer advantages in efficiency in those cases. ${ }^{84,85}$ Eully numerical calculations are also more conducive to parallelization, ${ }^{85}$ an advantage that becomes more important as the system size increases. Numerical differentiation is required for SCTST calculations even when analytical first or second derivatives are available, 66,67 and the approach discussed in this study, which uses Richardson extrapolation, ${ }^{86}$ enables systematic improvement of the accuracy of these numerical derivatives. Their accuracy can be adjusted according to what is required for the system being investigated.

FD and 1-D calculations are performed in this study for the following reactions:

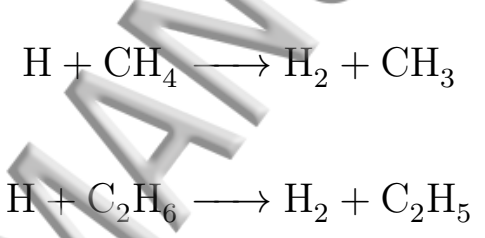

The size of these systems is small enough that FD SCTST calculations are computationally tractable but large enough to allow evaluation of the impact on calculated rate constants of a significant reduction in the number of DOFs treated. There are a wide variety of existing theoretical and experimental studies on reaction (1), as it is the simplest $\mathrm{H}$ atom abstraction reaction involving a hydrocarbon. ${ }^{33,34,37,40,59,60,87-90}$ Reaction (2), although more complicated, has also been studied as a prototypical H atom abstraction reaction. ${ }^{91-94}$ Both reactions are important in combustion processes ${ }^{18,91}$ and involve the exchange of a light $\mathrm{H}$ atom, suggesting the importance of quantum mechanical effects in determining the reaction rate. Comparing results from SCTST to those from conventional TST will allow for an evaluation of the accuracy with which SCTST treats these effects.

The remainder of this paper is organized as follows. Section II discusses the methods employed to calculate rate constants from quartic force fields and the application of Richardson extrapolation to quartic force field calculations. Section III presents the results of FD and 1-D calculations on reactions (1) and (2). Finally, Section IV summarizes the key findings of this study. 


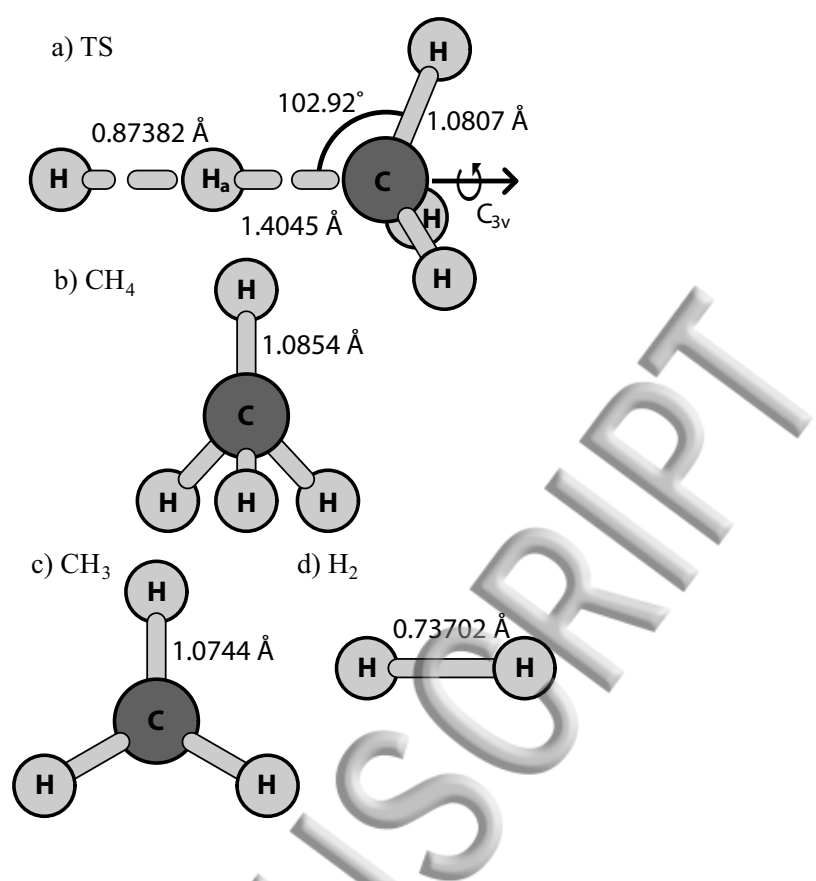

FIG. 1. Optimized structures of the stationary points for reaction (1), i.e., (a) the TS, (b) $\mathrm{CH}_{4}$, (c) $\mathrm{CH}_{3}$, and (d) $\mathrm{H}_{2}$, calculated at the MP2/cc-pVTZ level of theory. Bond lengths are reported in $\AA$, and bond angles in degrees. In (a), the $\mathrm{H}$ atom being abstracted from $\mathrm{CH}_{4}$ is denoted as $\mathrm{H}_{\mathrm{a}}$.

\section{METHODS}

\section{A. Ab Initio Calculations}

All quantum chemistry calculations in this study were performed using the MOLPRO software package. ${ }^{7}$ The geometries of the stationary points on the PES were found using second-order Møller-Plesset perturbation theory (MP2) with a correlation consistent polarized valence triple- $\zeta$ Dunning basis set $^{95}$ (cc-pVTZ). A tight geometry optimization condition (maximum gradient component less than $10^{-8}$ Eh $a_{0}^{-1}$ ) was used to calculate the transition state (TS) geometries, as suggested by Barone ${ }^{73}$ for the purpose of anharmonic constant calculations. No symmetry constraints were applied in geometry optimization calculations in order to ensure that no species had strictly degenerate frequencies. Barone ${ }^{73}$ suggested that degeneracy be broken in order to allow the use of formulas for asymmetric tops in anharmonic constant calculations, which greatly simplifies SCTST calculations. Recent SCTST studies ${ }^{66,67}$ employed the MultiWell Program Suite ${ }^{96,97}$ to perform calculations, which only includes formulas for asymmetric tops, suggesting that degeneracy was broken in these studies as well. 

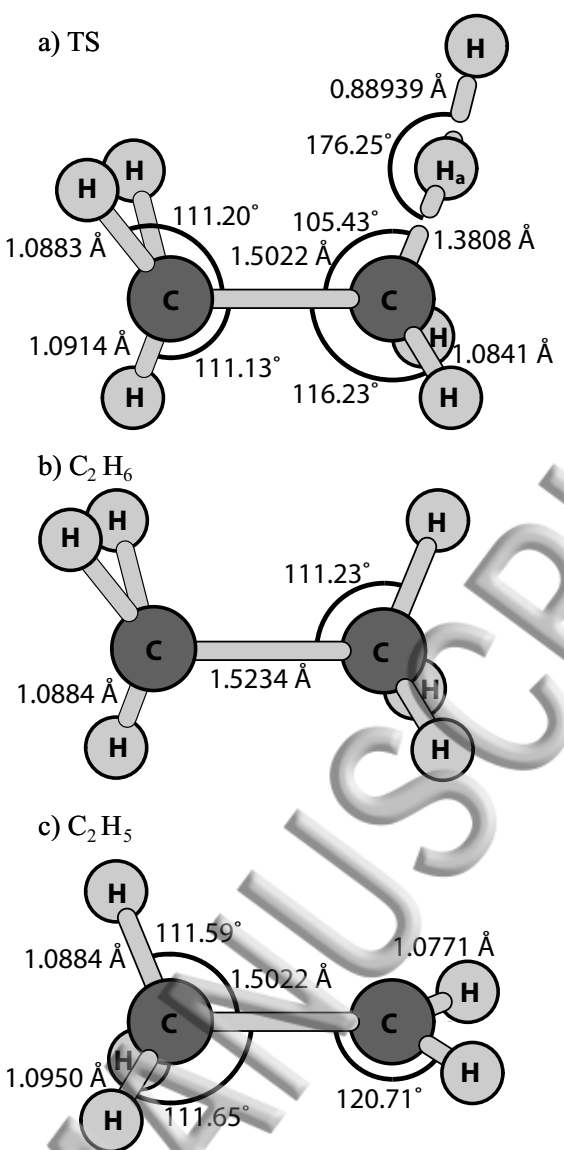

FIG. 2. Optimized structures of the stationary points for reaction (2), presented as in Fig. 1.

Geometries of the stationary points of reaction (1) are presented in Fig. 1. The $\mathrm{C}-\mathrm{H}$ and $\mathrm{C}-\mathrm{H}_{\mathrm{a}}$ bond lengths of the TS differ from those obtained in previous theoretical studies ${ }^{34,39}$ by $<1 \%$. The $\mathrm{H}-\mathrm{H}_{\text {a }}$ bond is approximately $2 \%$ shorter than those reported in the previous studies. Geometries of the reactants and products exhibit a similar level of agreement. Geometries for reaction (2) are presented in Fig. 2. The $\mathrm{H}-\mathrm{H}_{\mathrm{a}}$ bond in the TS is $3 \%$ longer than that calculated previously, ${ }^{91}$ and the $\mathrm{C}-\mathrm{H}_{\mathrm{a}}$ bond is $3 \%$ shorter. The remaining bond lengths and angles of the TS and other stationary points exhibit closer agreement.

Single-point energies of the stationary points at these geometries were obtained at the coupled cluster level including single, double, and perturbative triple excitations $[\mathrm{CCSD}(\mathrm{T})]^{98}$ using the augmented cc-pVTZ basis set. ${ }^{99}$ These results, as well as the reaction barrier heights, are presented in Table I. The nonadiabatic forward barrier height of reaction (1) is $0.439 \mathrm{kcal} \mathrm{mol}^{-1}$ greater and $0.6 \mathrm{kcal} \mathrm{mol}^{-1}$ less than those calculated at the CCSD(T)/ccpVTZ level of theory in Refs. 18 and 19, respectively. The forward barrier height of reac- 
ABLE I. Single-point CCSD(T)/aug-cc-pVTZ energies of the stationary points of reactions (1) and (2), and the associated forward and reverse barrier heights and reaction enthalpies.

\begin{tabular}{c|c|c}
\hline \hline \multicolumn{3}{|c}{ Molecular energies (Eh) } \\
\hline & $\mathrm{R}=\mathrm{CH}_{3}$ & $\mathrm{R}=\mathrm{C}_{2} \mathrm{H}_{5}$ \\
\hline $\mathrm{RH}$ & -40.44087814 & -79.67985655 \\
$\mathrm{H}$ & -0.49982118 & -0.49982118 \\
$\mathrm{TS}$ & -40.91725818 & -80.16078001 \\
$\mathrm{R}$ & -39.76359163 & -79.00787961 \\
$\mathrm{H}_{2}$ & -1.17231562 & -1.17231562 \\
\hline Reaction Energetics & $\left(\text { kcal mol }{ }^{-1}\right)^{a}$ \\
\hline & Reaction $(1)$ & Reaction $(2)$ \\
\hline$\Delta V_{f}$ & 14.710 & 11.859 \\
$\Delta V_{r}$ & 11.703 & 12.183 \\
$\Delta \Delta_{r} H$ & 3.007 & -0.325 \\
\hline \hline Zero-point energies are not included in these quantities.
\end{tabular}

tion (2) is $0.1 \mathrm{kcal} \mathrm{mol}^{-1}$ less than that reported in Ref. 19, which was calculated at the CCSD(T)-F12a/cc-pVTZ-F12a level of theory.

Hessian matrices, which were used to calculate anharmonic constants and vibrational partition functions, were calculated at the MP2/cc-pVTZ level of theory, which is commonly employed in rate constant calculations. ${ }^{19,46,100-102}$ All Hessian elements were calculated from central differences of the energy, using the NUMERICAL option in MOLPRO. Richardson extrapolation was used to improve the accuracy of Hessian elements for the TS, as described in the following section. This involves calculating the numerical Hessian using multiple step sizes by changing the STEP parameter in MOLPRO.

\section{B. Numerical Differentiation by Richardson Extrapolation}

In general terms, Richardson extrapolation is a method for accelerating convergence in a calculation of the value of an infinite series. ${ }^{103}$ Ridders ${ }^{86}$ adapted this technique to the calculation of the derivatives of a function. ${ }^{104}$ Calculations of a numerical derivative at larger 
Publishintgp sizes are used to remove the contribution of higher-order derivatives to the error in a calculation at a smaller step size. A summary of this method is presented below.

Consider a Taylor expansion of the function $f$ about a point $x$, evaluated at $(x+h)$ and $(x-h):$

$$
\begin{gathered}
f(x+h)=f(x)+f^{\prime}(x) h+\sum_{n=2}^{\infty} \frac{1}{n !} f^{(n)}(x) h^{n} \\
f(x-h)=f(x)-f^{\prime}(x) h+\sum_{n=2}^{\infty} \frac{(-1)^{n}}{n !} f^{(n)}(x) h^{n}
\end{gathered}
$$

where $f^{(n)}(x)$ denotes the $n^{\text {th }}$ derivative of $f$ evaluated at $x$. Combining these expressions gives:

$$
A^{(0)}(h) \equiv \frac{f(x+h)-f(x-h)}{2 h}=f^{\prime}(x)+\sum_{n=1}^{\infty} \frac{1}{(2 n+1) !} f^{(2 n+1)}(x) h^{2 n}
$$

Only odd-numbered derivatives of $f$ are included, and these error terms are proportional to only even powers of $h$.

Next considering calculations of this derivative with a different step size, $A^{(0)}(c h)$, where $c>1$, gives:

$$
A^{(0)}(c h)=\frac{f(x+c h)-f(x-c h)}{2 c h}=f^{\prime}(x)+\sum_{n=1}^{\infty} \frac{1}{(2 n+1) !} f^{(2 n+1)}(x) c^{2 n} h^{2 n}
$$

Both $A^{(0)}(h)$ and $A^{(0)}(c h)$ represent the exact value of the first derivative, $f^{\prime}(x)$, plus an infinite sum of error terms. They can be combined as follows:

$$
\frac{c^{2} A^{(0)}(h)-A^{(0)}(c h)}{c^{2}-1}=f^{\prime}(x)+\sum_{n=1}^{\infty} \frac{1}{(2 n+1) !} f^{(2 n+1)}(x) \frac{c^{2}-c^{2 n}}{c^{2}-1} h^{2 n}
$$

When $n=1$, the first term in the above sum is 0 . Thus, this expression can be rewritten:

$$
\frac{c^{2} A^{(0)}(h)-A^{(0)}(c h)}{c^{2}-1}=f^{\prime}(x)+\sum_{n=2}^{\infty} \frac{1}{(2 n+1) !} f^{(2 n+1)}(x) \frac{c^{2}-c^{2 n}}{c^{2}-1} h^{2 n}
$$

In situations in which this formulation is useful, successively higher-order terms in the Taylor expansion constitute lesser contributions to $f(x)$. The left-hand side of the above expression will be referred to as a first-order extrapolation of $f^{\prime}(x)$. Because it does not include the $f^{(3)}(x)$ term from the Taylor expansion, it is an estimate of $f^{\prime}(x)$ with a lesser error than the zeroth-order extrapolation (Eq. 5).

The key feature of Eq. 5 that enables the construction of an expression for $f^{\prime}(x)$ with fewer error terms is the dependence of the error terms on even powers of $h$. Eq. 8 also 
Publishidgends only on even powers of $h$, so the above technique can be applied again to further reduce the error. Defining Eq. 8 as $B(h)$, we have:

$$
\frac{c^{4} B(h)-B(c h)}{c^{4}-1}=f^{\prime}(x)+\sum_{n=3}^{\infty} \frac{1}{(2 n+1) !} f^{(2 n+1)}(x) \frac{c^{2}-c^{2 n}}{c^{2}-1} \frac{c^{4}-c^{2 n}}{c^{4}-1} h^{2 n}
$$

where the error is further reduced because the above sum does not include the $f^{(5)}(x)$ term. Substituting the definition of $B(h)$ and simplifying gives:

$$
\frac{c^{4} B(h)-B(c h)}{c^{4}-1}=\frac{c^{6} A(h)-\left(c^{2}+c^{4}\right) A(c h)+A\left(c^{2} h\right)}{\left(c^{2}-1\right)^{2}\left(c^{2}+1\right)}
$$

This is a second-order extrapolation of $f^{\prime}(x)$, and evaluating it involves calculating numerical derivatives at three different step sizes. In theory, the above approach can be applied arbitrarily many times, with successively larger step sizes $c^{n} h$, with $n$ a positive integer, to reduce the error in a numerical calculation of $f^{\prime}(x)$.

Richardson extrapolation can also be used to improve estimates of second derivatives as follows:

$$
\frac{f(x+h)-2 f(x)+f(x-h)}{h^{2}}=f^{\prime \prime}(x)+\sum_{n=1}^{\infty} \frac{2}{(2 n+2) !} f^{(2 n+2)}(x) h^{2 n}
$$

Because the error terms are proportional to even powers of $h$, successively better approximations of $f^{\prime \prime}(x)$ can be obtained from numerical calculations of $f^{\prime \prime}(x)$ with step sizes $c^{n} h$, as above. Expressions for the first-and second-order extrapolations of the derivative are identical to those given above, except that $A(h)$ is defined as $f^{\prime \prime}(x)$ calculated numerically with a step size of $h$.

Furthermore, this technique can be applied to calculate mixed second partial derivatives numerically. Briefly, the function $f(x, y)$ is expanded in four Taylor series at $f(x \pm h, y \pm h)$. These expressions are combined to give:

$$
\begin{aligned}
& \frac{f(x+h, y+h)-f(x+h, y-h)-f(x-h, y+h)+f(x-h, y-h)}{4 h^{2}}= \\
& f_{x y}(x, y)+\sum_{n_{x}=2}^{\infty} \sum_{n_{y}=2}^{\infty} \frac{1}{n_{x} ! n_{y} !}\left(\frac{\partial^{\left(n_{x}+n_{y}\right)} f}{\partial x^{n_{x}} \partial y^{n_{y}}}\right) h^{n_{x}+n_{y}}\left[1-(-1)^{n_{x}}-(-1)^{n_{y}}+(-1)^{n_{x}+n_{y}}\right]
\end{aligned}
$$

which again is the numerical expression for the mixed second partial derivative of $f$ with respect to $x$ and $y$, here denoted $f_{x y}(x, y)$, plus a sum of error terms. The error terms are nonzero only when both $n_{x}$ and $n_{y}$ are odd, in which case the quantity $\left(n_{x}+n_{y}\right)$ is even, so all error terms are proportional to even powers of $h$. The analysis discussed above can therefore be applied here, yielding analogous expressions for higher-order extrapolations of the derivative. 
The quartic force fields used in SCTST are most conveniently defined in terms of second, third, and fourth directional derivatives of the system's potential along normal mode eigenvectors. ${ }^{63,64,105}$ These derivatives are calculated according to the commonly employed technique referenced in Section I. ${ }^{71,73,106}$ First, $\mathbf{H}(0)$ is defined as the Hessian matrix evaluated at the stationary point of the potential (i.e., the minimum)for a molecule and the saddle point for the TS), and the elements of the matrix $\mathbf{M}$ are defined as follows:

$$
M_{i j}=\delta_{i j} m_{i}^{-1 / 2}
$$

where the atomic masses $m_{i}$ correspond to the Cartesian coordinates used to construct the Hessian matrix. The mass-weighted Hessian matrix, $\mathbf{M H}(0) \mathbf{M}$, is diagonalized to obtain a matrix of eigenvectors, L. The matrix $\boldsymbol{\Phi}(0)$ is defined as follows:

$$
\Phi(0)=\mathbf{L}^{\mathrm{T}} \mathbf{M H}(0) \mathbf{M L}
$$

such that its elements are directional derivatives of the potential along normal mode eigenvectors:

$$
\phi_{i j}(0)=\frac{\partial^{2} V}{\partial Q_{i} \partial Q_{j}}
$$

By definition, $\boldsymbol{\Phi}(0)$ is diagonal, and its elements are proportional to the squares of the system's harmonic frequencies. These elements can be differentiated to obtain third and fourth directional-derivatives of the potential at the stationary point. Third derivatives, $f_{i j k}$, are calculated as follows:

$$
f_{i j k}=\frac{\partial^{3} V}{\partial Q_{i} \partial Q_{j} \partial Q_{k}}=\frac{1}{3}\left(\frac{\partial \phi_{j k}(0)}{\partial Q_{i}}+\frac{\partial \phi_{k i}(0)}{\partial Q_{j}}+\frac{\partial \phi_{i j}(0)}{\partial Q_{k}}\right)
$$

Note that $f_{i j k}$ can be obtained by differentiating three different matrix elements, and the above formula reflects an average of those three derivatives. Only fourth derivatives of the form $f_{i i j j}$ are needed for anharmonic constant calculations. ${ }^{64}$ These are calculated as follows:

$$
f_{i i j j}=\frac{1}{2}\left(\frac{\partial^{2} \phi_{i i}(0)}{\partial Q_{j}^{2}}+\frac{\partial^{2} \phi_{j j}(0)}{\partial Q_{i}^{2}}\right)
$$

In principle, these derivatives could also be obtained by differentiating the matrix element $\phi_{i j}(0)$ along $Q_{i}$ and $Q_{j}$. However, as will be made apparent below, adding these terms in 
Publishing calculation of these derivatives would greatly increase the number of Hessian matrix calculations required for the implementation presented in this study.

Calculating these derivatives numerically involves obtaining the Hessian matrix $\mathbf{H}\left( \pm \Delta Q_{i}\right)$ at positive and negative displacements $\pm \Delta Q_{i}$ away from the stationary point along each respective eigenvector. If $\mathbf{x}(0)$ represents the vector of atomic coordinates at the stationary point, then $\mathbf{x}\left( \pm \Delta Q_{i}\right)$, the coordinates at the displaced geometry, is calculated as follows:

$$
\mathbf{x}\left( \pm \Delta Q_{i}\right)=\mathbf{x}(0) \pm \Delta Q_{i} \mathbf{M L}_{i}
$$

where $\mathbf{L}_{i}$ denotes the $i^{\text {th }}$ eigenvector. The matrix $\boldsymbol{\Phi}\left( \pm \Delta Q_{i}\right)$ is thus defined at these displaced geometries:

$$
\boldsymbol{\Phi}\left( \pm \Delta Q_{i}\right)=\mathbf{L}^{\mathrm{T}} \mathbf{M H}\left( \pm \Delta Q_{i}\right) \mathbf{M L}
$$

$\boldsymbol{\Phi}\left( \pm \Delta Q_{i}\right)$ is not necessarily diagonal because the matrix of eigenvectors $\mathbf{L}$ diagonalizes the mass-weighted Hessian matrix at the stationary point, and not at the displaced geometries. The derivatives $f_{i j k}$ can then be calculated numerically as follows: ${ }^{71,73}$

$$
f_{i j k}=\frac{1}{3}\left(\frac{\boldsymbol{\Phi}_{j k}\left(\Delta Q_{i}\right)-\boldsymbol{\Phi}_{j k}\left(-\Delta Q_{i}\right)}{2 \Delta Q_{i}}+\frac{\boldsymbol{\Phi}_{k i}\left(\Delta Q_{j}\right)-\boldsymbol{\Phi}_{k i}\left(-\Delta Q_{j}\right)}{2 \Delta Q_{j}}+\frac{\boldsymbol{\Phi}_{i j}\left(\Delta Q_{k}\right)-\boldsymbol{\Phi}_{i j}\left(-\Delta Q_{k}\right)}{2 \Delta Q_{k}}\right)
$$

$f_{i i j j}$ is calculated similarly:

$$
f_{i i j j}=\frac{1}{2}\left(\frac{\boldsymbol{\Phi}_{i i}\left(\Delta Q_{j}\right)+\boldsymbol{\Phi}_{i i}\left(-\Delta Q_{j}\right)-2 \boldsymbol{\Phi}_{i i}(0)}{\Delta Q_{j}^{2}}+\frac{\boldsymbol{\Phi}_{j j}\left(\Delta Q_{i}\right)+\boldsymbol{\Phi}_{j j}\left(-\Delta Q_{i}\right)-2 \boldsymbol{\Phi}_{j j}(0)}{\Delta Q_{i}^{2}}\right)
$$

Eqs. 20 and 21 comprise numerical derivatives of elements of $\boldsymbol{\Phi}$; elements of the Hessian matrices used to calculate $\mathbf{\Phi}$ are also calculated numerically. Thus, Richardson extrapolation can be applied in two independent ways to calculations of the potential derivatives $f_{i j k}$ and $f_{i i j j}$. Calculations can be performed with higher-order extrapolations of Hessian elements and zeroth-order extrapolations of derivatives of elements of $\boldsymbol{\Phi}$, or vice-versa, or higher-order extrapolations of both.

Displacements along normal mode eigenvectors are given in dimensionless reduced normal coordinates. A displacement $\Delta Q_{i}$ in units of (length mass ${ }^{1 / 2}$ ) is calculated from a displacement $\Delta q$ in reduced normal coordinates as follows: ${ }^{73,107,108}$

$$
\Delta Q_{i}=\frac{\hbar^{1 / 2}}{\omega_{i}^{1 / 2}} \Delta q
$$

where $\omega_{i}$ is the frequency of the $i^{\text {th }}$ normal mode. In this study, Richardson extrapolation is applied to both Hessian elements and to the potential derivatives $f_{i j k}$ and $f_{i i j j}$ for the 
Publishings of reactions (1) and (2). Richardson extrapolation is not applied to the calculation of anharmonic constants for the reactants $\mathrm{CH}_{4}$ and $\mathrm{C}_{2} \mathrm{H}_{6}$, as those calculated with Richardson extrapolation were not found to differ significantly from those calculated without it. A single displacement of $\Delta q=0.4$ was used.

The anharmonic constants $x_{i j}$ and associated constant term $G_{0}$ can then be calculated from the third and fourth derivatives according to second-order vibrational perturbation theory (VPT2): $:^{63,64,105}$

$$
\begin{gathered}
x_{i i}=\frac{\hbar^{2}}{16 \omega_{i}^{2}}\left(f_{i i i i}-\sum_{k=1}^{F} \frac{f_{i i k}^{2} \frac{8 \omega_{i}^{2}-3 \omega_{k}^{2}}{\omega_{k}^{2}}}{4 \omega_{i}^{2}-\omega_{k}^{2}}\right) \\
x_{i k}=\frac{\hbar^{2}}{4 \omega_{i} \omega_{k}}\left(f_{i i k k}-\sum_{j=1}^{F} \frac{f_{i i j} f_{j k k}}{\omega_{j}^{2}}+\sum_{j=1}^{F} \frac{2 f_{i i k}^{2}\left(\omega_{i}^{2}+\omega_{k}^{2}-\omega_{i}^{2}\right)}{\left[\left(\omega_{i}+\omega_{k}\right)^{2}-\omega_{i}^{2}\right]\left[\left(\omega_{i}-\omega_{k}\right)^{2}-\omega_{i}^{2}\right]}\right)+ \\
\left(\frac{\omega_{i}}{\omega_{k}}+\frac{\omega_{k}}{\omega_{i}}\right) \sum_{\alpha} B_{\alpha}\left(\zeta_{i k}^{\alpha}\right)^{2}, i \neq k \\
G_{0}=\frac{\hbar^{2}}{64} \sum_{i=1}^{F} \frac{f_{i i i i}}{\omega_{i}^{2}}-\frac{7 \hbar^{2}}{576} \sum_{i=1}^{F} \frac{f_{i i i}^{2}}{\omega_{i}^{2}}+\frac{3 \hbar^{2}}{64} \sum_{i \neq k}^{\frac{f_{i i k}^{2}}{\left(4 \omega_{i}^{2}-\omega_{k}^{2}\right) \omega_{i}^{2}}} \\
-\frac{\hbar^{2}}{4} \sum_{i<j<k} \frac{f_{i j k}^{2}}{\left[\left(\omega_{i}+\omega_{k}\right)^{2}-\omega_{j}^{2}\right]\left[\left(\omega_{i}-\omega_{k}\right)^{2}-\omega_{j}^{2}\right]}
\end{gathered}
$$

where $B_{\alpha}$ is the rotational constant associated with axis $\alpha$, and $\zeta_{i k}^{\alpha}$ is the associated Coriolis coupling constant/calculated as described in Ref. 109. Fermi resonances in these formulas were deperturbed as described previously. ${ }^{73}$

\section{Density of States}

The partition functions required for rate constant calculations are typically calculated from a Boltzmann-weighted sum over energy levels. The anharmonic energy levels of a species are given according to VPT2: ${ }^{63,64,105}$

$$
E_{\{n\}}=\sum_{i=1}^{F} \hbar \omega_{i}\left(n_{i}+1 / 2\right)+\sum_{i \geq j} x_{i j}\left(n_{i}+1 / 2\right)\left(n_{j}+1 / 2\right)+G_{0}
$$

where $\{n\}$ is the set of quantum numbers of the $F$ vibrational modes of the species. As this expression for the energy is not separable, it is not possible to separate the partition 
Publishifngiction for this system into a product of individual partition functions for each mode, as is the case for a system of harmonic oscillators. Consequently, one must enumerate over all configurations of $\{n\}$. This is very computationally expensive for systems with more than 3 or 4 atoms. .110 $^{110}$

To improve the efficiency of this calculation for larger systems, Nguyen and Barker ${ }^{110}$ adapted a random walk algorithm in energy space developed-originally by Wang and Landau ${ }^{111,112}$ and modified later by Basire et al. ${ }^{113}$ Details of this Wang-Landau algorithm can be found in Ref. 110. Briefly, the energy space from the anharmonic zero-point energy of the species to a maximum energy $E_{\max }$, chosen to ensure convergence of the partition function, is partitioned into energy bins, and each bin is initialized with a trial density of states. A density-weighted Monte-Carto sampling of the configurations $\{n\}$ is then performed, and the density of states in each bin is modified as different bins are visited. This yields a final density of states $\rho(E)$. The Boltzmann-weighted average of $\rho(E)$ is the anharmonic partition function $Q_{\mathrm{anh}}$ :

$$
Q_{\operatorname{anh}}(T)=\int_{0}^{E_{\max }} \rho(E) \exp \left(-E k_{B}^{-1} T^{-1}\right) \mathrm{d} E
$$

In this study, parameters of $N_{\text {iter }}=22$ and $N_{\text {trial }}=10^{7}$, defined in Ref. 110, were used. This choice of parameters has previously been demonstrated to be sufficient for convergence. ${ }^{110}$ For the species considered in this study, our implementation of the Wang-Landau algorithm required less than $600 \mathrm{~s}$ of $\mathrm{CPU}$ time on a $2.4 \mathrm{GHz}$ processor.

\section{E. FD SCTST Rate Constants}

In FD SCTST, as in other rate constant theories, the rate constant $k_{\mathrm{FD}}(T)$ is calculated from a Boltzmann-weighted average of the cumulative reaction probability (CRP), $N_{\mathrm{FD}}\left(E_{\mathrm{v}}\right): 63,67,68$

$$
k_{\mathrm{FD}}(T)=\frac{1}{h} \frac{Q_{\mathrm{trans}}^{\ddagger}(T) Q_{\mathrm{rot}}^{\ddagger}(T) Q_{\text {elec }}^{\ddagger}(T) \int_{0}^{\infty} N_{\mathrm{FD}}\left(E_{\mathrm{v}}\right) \exp \left(-E_{\mathrm{v}} k_{B}^{-1} T^{-1}\right) \mathrm{d} E_{\mathrm{v}}}{Q_{\mathrm{r}}(T)}
$$

where $Q_{\mathrm{r}}(T)$ is the partition function for the reactants, and $Q_{\text {trans }}^{\ddagger}(T), Q_{\text {rot }}^{\ddagger}(T)$, and $Q_{\text {elec }}^{\ddagger}(T)$ are the translational, rotational, and electronic partition functions for the transition state. Rotational partition functions were calculated from equilibrium geometries obtained from $a b$ initio calculations, and electronic partition functions were assumed to be 1 and 2 for closed- 
Publishiargd open-shell systems, respectively. Anharmonic partition functions were calculated for the vibrational DOFs of the reactants as described above.

Both $\mathrm{C}_{2} \mathrm{H}_{6}$ and the TS of reaction (2) have a normal mode that corresponds to torsional motion about the $\mathrm{C}-\mathrm{C}$ bond. These torsional modes generally have low frequencies, so treating them with a harmonic or quartic force field can be problematic. Consequently, these modes were not considered vibrational DOFs and therefore not included in anharmonic constant calculations or anharmonic partition functions. Instead, they were treated with partition functions for hindered rotors using the "single-frequency" approximation presented in Ref. 114. Thus, the expression for the FD rate constant for reaction (2) has an additional partition function in the numerator and in the denominator.

The $F$ vibrational modes of the TS are considered in a FD SCTST calculation. One of these modes, the reaction mode, has an imaginary frequency and is tangent to the reaction path at the TS. The remaining $F-1$ bound modes have real frequencies and an associated set of quantum numbers $\{n\}^{\prime}$. Typically, the semiclassical CRP is calculated as a sum of state-dependent reaction probabilities, $P_{\{n\}^{\prime}}\left(E_{\mathrm{v}}\right)^{: 63,67,68}$

$$
N_{\mathrm{FD}}\left(E_{\mathrm{v}}\right)=\sum_{\{n\}^{\prime}} P_{\{n\}^{\prime}}\left(E_{\mathrm{v}}\right)
$$

where the sum is over all energetically allowed configurations of $\{n\}^{\prime}$ at energy $E_{\mathrm{v}}$. The maximum allowed quantum number for a particular mode, $n_{\max , i}$, is a function of $E_{\mathrm{v}}$ and the quantum numbers of all other bound modes: ${ }^{110}$

$$
n_{\max , i}= \begin{cases}n_{D, i} & \text { if } E_{\mathrm{v}}-E_{F-2} \geq D_{i} \\ n_{D, i}\left[1-\left(1-E_{\mathrm{v}} D_{i}^{-1}\right)^{1 / 2}\right] & \text { if } E_{\mathrm{v}}-E_{F-2}<D_{i}\end{cases}
$$

where $E_{F-2}$ is the vibrational energy calculated from the $F-2$ quantum numbers of the other bound modes (Eq. 26), and

$$
\begin{aligned}
n_{D, i} & =-\frac{\hbar \omega_{i}+\sum_{j \neq i} x_{i j}\left(n_{j}+1 / 2\right)}{2 x_{i i}}-\frac{1}{2} \\
D_{i} & =-\frac{\left[\hbar \omega_{i}+\sum_{j \neq i} x_{i j}\left(n_{j}+1 / 2\right)\right]^{2}}{4 x_{i i}}
\end{aligned}
$$

Each state-dependent reaction probability is calculated from a barrier penetration integral $\theta_{\{n\}^{\prime}}$, which is calculated analytically according to the method described by Wagner. ${ }^{70}$ 
PublishiRgeviously, we found that this method yields results of comparable or better accuracy to those obtained from SCTST in its original form, as it treats tunneling at low energies close to the reaction's quantum mechanical threshold energy more accurately. ${ }^{68,69}$ Briefly, this method involves constructing an asymmetric piecewise-continuous Eckart potential $V(s)$ from the reaction's forward and reverse barrier heights, the anharmonic constant $x_{F F}$, and an effective reaction mode frequency $\Omega_{\{n\}^{\prime}}$, defined as follows:

$$
\Omega_{\{n\}^{\prime}}=\operatorname{Im}\left(\hbar \omega_{F}\right)+\sum_{i=1}^{F-1} \operatorname{Im}\left(x_{i F}\right)\left(n_{i}+1 / 2\right)
$$

where the index $F$ indicates the reaction mode. Treating $\Omega_{\{n\}^{\prime}}$ as the reaction-mode frequency accounts for coupling between the bound modes and the reaction mode for a particular configuration $\{n\}^{\prime}$. The barrier penetration integral is then calculated according to WKB Theory: ${ }^{115}$

$$
\theta_{\{n\}^{\prime}}\left(E_{\mathrm{v}}\right)=\frac{1}{\hbar} \int\left[2\left(V(s)-E_{\mathrm{v}}+E_{\{n\}^{\prime}}+G_{0}\right)\right]^{1 / 2} d s
$$

where $s$ is the reaction coordinate, with units of (mass ${ }^{1 / 2}$ length), and $E_{\{n\}^{\prime}}$ is the energy in the $F-1$ bound modes, calculated from Eq. 26. $G_{0}$ was defined in Eq. 22. Finally, the state-dependent reaction probability is calculated as follows: ${ }^{41}$

$$
P_{\{n\}^{\prime}}\left(E_{\mathrm{v}}\right)= \begin{cases}\left\{1+\exp \left[2 \theta_{\{n\}^{\prime}}\left(E_{\mathrm{v}}\right)\right]\right\}^{-1} & \text { if } E_{\mathrm{v}}-E_{\{n\}^{\prime}}<\Delta V_{f}+G_{0}, \\ 1-P_{\{n\}^{\prime}}\left(2 \Delta V_{f}-E_{\mathrm{v}}\right) & \text { if } \Delta V_{f}+G_{0}<E_{\mathrm{v}}-E_{\{n\}^{\prime}} \leq \\ 1 & \Delta V_{f}+2 G_{0}+\min \left(\Delta V_{f}, \Delta V_{r}\right)+E_{\{n\}^{\prime}} \\ & \text { if } \Delta V_{f}+2 G_{0}+\min \left(\Delta V_{f}, \Delta V_{r}\right) \\ & +E_{\{n\}^{\prime}}<E_{\mathrm{v}}-E_{\{n\}^{\prime}} .\end{cases}
$$

where $\Delta V_{f}$ and $\Delta V_{r}$ are the reaction's forward and reverse barrier heights, respectively. The zero-point energies (ZPE) of the reactants and products are included in $\Delta V_{f}$ and $\Delta V_{r}$, respectively, but the ZPE of the TS is not, as it is included in the expression for $E_{\{n\}^{\prime}}$ (Eq. 26).

Typically, the rate constant expression in Eq. 28 is evaluated by enumerating over all configurations $\{n\}^{\prime}$ with energies $E_{\{n\}^{\prime}}$ less than a maximum energy $E_{\text {max }}$ chosen to ensure convergence of the integral. As is the case when evaluating partition functions, this is impractical for systems with more than 3 or 4 atoms. The Wang-Landau algorithm ${ }^{110}$ is 
Publishing efore applied to approximate the density of vibrational states of the TS, $\rho_{\mathrm{TS}}(E)$. Average values of $\Omega_{\{n\}^{\prime}}$ and $E_{\{n\}^{\prime}}$ are calculated for each energy bin from the configurations of $\{n\}^{\prime}$ sampled within that bin. ${ }^{96,97}$ The average reaction probability for the $i^{\text {th }}$ bin, $P_{i}(E)$, is calculated from these parameters according to Eqs. 34 and 35. Thus, the CRP is calculated as follows: ${ }^{97}$

$$
N_{\mathrm{FD}}(E)=\sum_{i=1}^{E / \Delta E} \rho_{\mathrm{TS}}\left(E_{i}\right) P_{i}(E) \Delta E
$$

where $E_{i}$ is the energy of the $i^{\text {th }}$ bin and $\Delta E$ is the bin width.

\section{F. 1-D SCTST Rate Constants}

In a 1-D SCTST calculation, only the reaction mode is treated anharmonically. The remaining $F-1$ spectator modes are treated harmonically and assumed not to couple with the reaction mode. Third and fourth derivatives of the potential with respect to spectator modes are assumed to be 0 . The anharmonic constant for the reaction mode is given as follows:

$$
x_{F F}=\frac{\hbar^{2}}{16 \omega_{F}^{2}}\left(f_{F F F F}-\frac{5 f_{F F F}^{2}}{3 \omega_{F}^{2}}\right)
$$

and the remaining anharmonic constants are 0 . The $G_{0}$ term is:

$$
G_{0}=\hbar^{2}\left(\frac{1}{64} \frac{f_{F F F F}}{\omega_{F}^{2}}-\frac{5}{576} \frac{f_{F F F}^{2}}{\omega_{F}^{4}}\right)
$$

The effective reaction mode frequency does not depend on the quantum numbers of the spectator modes:

$$
\Omega=\operatorname{Im}\left(\hbar \omega_{F}\right)
$$

The calculation of the CRP $N_{1-\mathrm{D}}$ is greatly simplified by neglecting the energy in the spectator modes when calculating the barrier penetration integral and instead including an additional partition function, $Q_{\text {spec }}^{\ddagger}(T)$ in the rate constant expression: ${ }^{18,102,116}$

$$
k_{\mathrm{RD}}(T)=\frac{1}{h} \frac{Q_{\mathrm{trans}}^{\ddagger}(T) Q_{\mathrm{rot}}^{\ddagger}(T) Q_{\mathrm{elec}}^{\ddagger}(T) Q_{\mathrm{spec}}^{\ddagger}(T) \int_{0}^{\infty} N_{1-\mathrm{D}}\left(E_{\mathrm{v}}\right) \exp \left(-E_{\mathrm{v}} k_{B}^{-1} T^{-1}\right) \mathrm{d} E_{\mathrm{v}}}{Q_{\mathrm{r}}(T)}
$$

$Q_{\mathrm{spec}}^{\ddagger}(T)$ is calculated from a product of individual harmonic oscillator partition functions for each spectator mode. This removes the need to enumerate over configurations $\{n\}^{\prime}$ when calculating the CRP. Only one asymmetric Eckart potential is constructed. 
ABLE II. MP2/cc-pVTZ harmonic frequencies of the stationary points of reaction (1), obtained with different orders of Richardson extrapolation applied to the calculation of Hessian matrix elements.

\begin{tabular}{|c|c|c|c|c|c|c|}
\hline \multirow[b]{2}{*}{ Order $^{a}$} & \multicolumn{2}{|c|}{$\mathrm{CH}_{4}$} & \multicolumn{3}{|c|}{$\mathrm{TS}$} & $\mathrm{CH}_{3}$ \\
\hline & $2^{\text {nd }}$ & $0^{\text {th }}$ & $4^{\text {th }}$ & $2^{\text {nd }}$ & $0^{\text {th }}$ & $0^{\text {th }}$ \\
\hline & 3211.83 & 3211.86 & $1642.93 i$ & $1643.02 i$ & $1644.09 i$ & 3368.94 \\
\hline & 3211.79 & 3211.76 & 3291.55 & 3291.51 & 3290.98 & 3368.73 \\
\hline & 3211.79 & 3211.70 & 3290.09 & 3290.08 & 3289.88 & 178 \\
\hline & 3076.09 & 3076.05 & 3133.46 & 3133.37 & 3132.10 & 04 \\
\hline & 1586.14 & 1586.14 & 1948.13 & 1948.02 & 1946 & 5.00 \\
\hline & 1586.13 & 1586.10 & 1473.15 & 1472.92 & 1468 & 186.593 \\
\hline & 1349.61 & 1349.61 & 1469.65 & 1460 & & \\
\hline & 1349.57 & 1349.59 & 1151.75 & & & \\
\hline & 1349.57 & 1349.56 & 1148.09 & & 144.02 & \\
\hline $\mathrm{ZPE}^{b}$ & 28.495 & 28.495 & 27.397 & 27.394 & 27.336 & 19.006 \\
\hline
\end{tabular}

$\overline{\bar{a} \text { The order of Richardson extrapolation applied to the calculation of the Hessian matrix ele- }}$ ments used to calculate frequencies. For all orders, the smallest step size used was $0.01 a_{0}$. ${ }^{b}$ Harmonic zero-point energy in $\mathrm{kcal} \mathrm{mol}^{-1}$.

\section{RESULTS AND DISCUSSION}

\section{A. Vibrational Frequencies}

Vibrational frequencies for the stationary points of reaction (1) are presented in Table II. Higher orders of Richardson extrapolation were applied to calculate Hessian elements and frequencies of $\mathrm{CH}_{4}$ and the transition state (TS). Step sizes of 0.01, 0.02, and $0.04 a_{0}$ were used for $\mathrm{CH}_{4}$, and step sizes from 0.01 to $0.16 a_{0}$ were used for the TS. All zeroth order calculations were performed at $0.01 a_{0}$. For $\mathrm{CH}_{4}$, second-order frequencies differ from their 
TABLE III. MP2/cc-pVTZ harmonic frequencies of the stationary points of reaction (2) used in rate constant calculations.

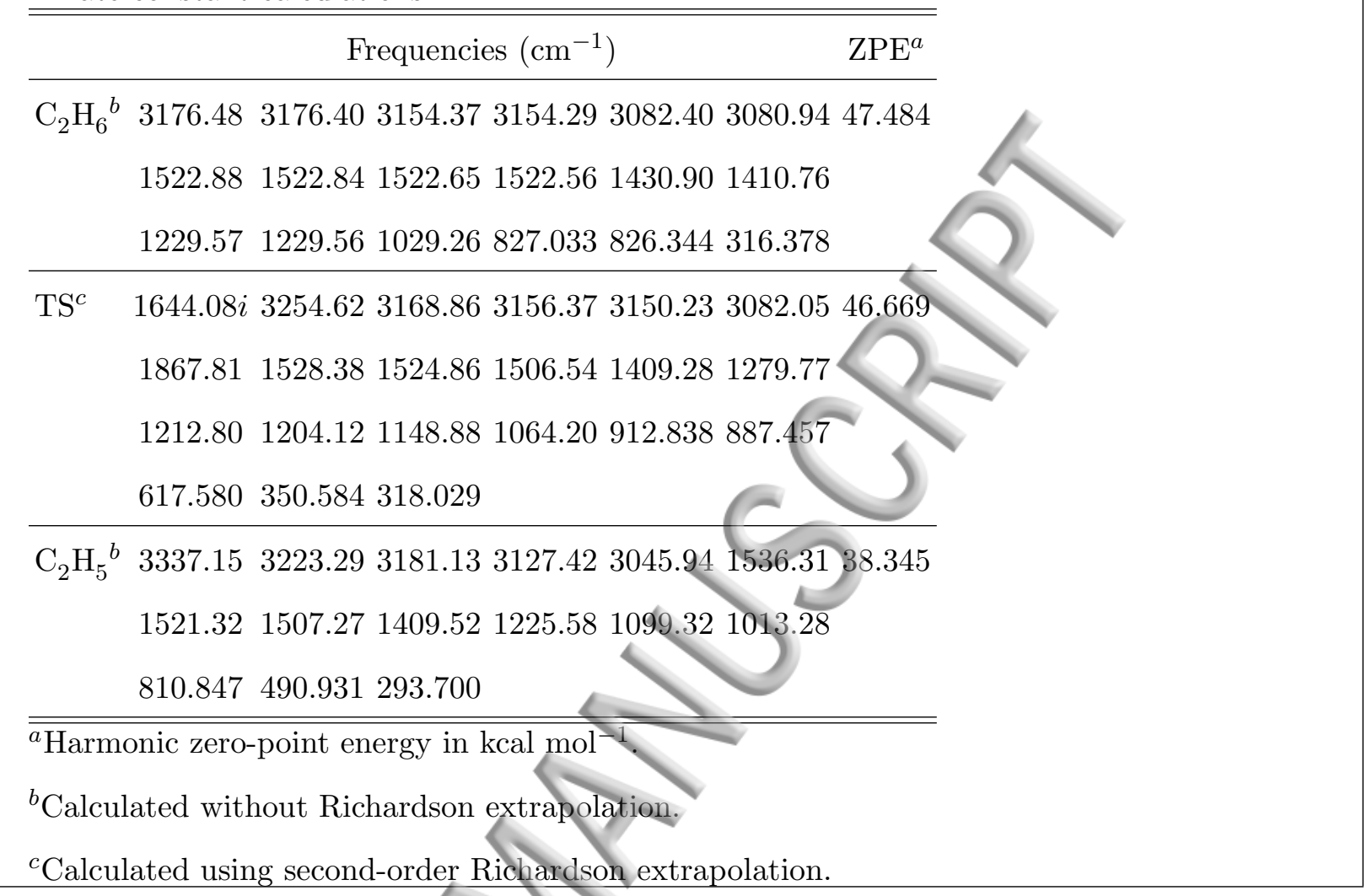

zeroth-order counterparts by at most $0.09 \mathrm{~cm}^{-1}$. Larger differences between second- and zeroth-order frequencies are observed for the TS, particularly for lower-frequency modes. The difference for the lowest-frequency mode of the TS is $7.516 \mathrm{~cm}^{-1}$. Fourth-order frequencies for the TS are significantly closer to second-order frequencies than to zeroth-order frequencies, indicating that Hessian elements converge as the order of extrapolation is increased. All frequencies exhibited agreement with those calculated previously by Banks et $a l .{ }^{18}$ at the same level of theory. The zeroth-order frequencies of the products, $\mathrm{CH}_{3}$ and $\mathrm{H}_{2}$ are also reported in Table II. These are used only to calculate the reaction's vibrationally adiabatic reverse barrier height, $\Delta V_{r}$ (Eq. 35). SCTST calculations are generally significantly less sensitive to the reverse barrier height than to the forward barrier height, so only zeroth-order frequencies were calculated for the products.

The vibrational frequencies used in rate constant calculations for reaction (2) are presented in Table III. Only second-order Richardson extrapolation was applied to the TS, as higher orders of extrapolation were not found to significantly change the frequencies for the 
Publishitig of reaction (1). The TS frequencies of reaction (2) differ somewhat from those reported in Ref. 91. This is likely due to the tighter geometry optimization condition used to calculate the TS geometry in this study and not to the application of Richardson extrapolation. Zeroth-order frequencies were more similar to second-order frequencies than to those from Ref. 91.

\section{B. Full-Dimensional SCTST Calculations}

\section{1. $\mathrm{H}+\mathrm{CH}_{4} \longrightarrow \mathrm{H}_{2}+\mathrm{CH}_{3}$ (reaction 1)}

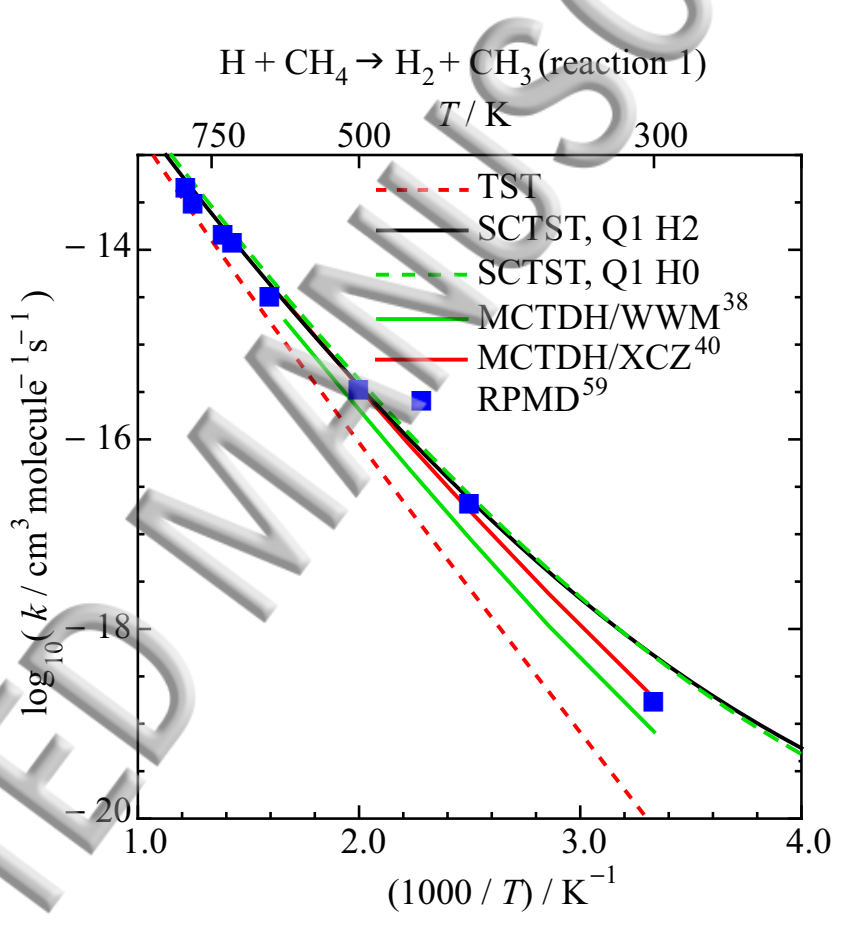

FIG. 3. Rate constants for reaction (1) calculated in this study by SCTST using two different sets of anharmonic constants ("Q1 H2," black curve, and "Q1 H0," dashed green curve), as defined in the text, presented in comparison with results from two previous MCTDH studies performed using the $\mathrm{WWM}^{38}$ and $\mathrm{XCZ}^{40}$ PESs (red and green curves, respectively), in addition to one RPMD study $^{59}$ (blue squares). Results from conventional Transition State Theory (TST) are presented for comparison (dashed red curve).

The anharmonic constants of the TS required for full-dimensional (FD) rate constant calculations on reaction (1) were calculated at three different levels of accuracy. At the 
Publishihigh hest level of accuracy, fourth-order Richardson extrapolation was applied to calculate elements of the TS Hessian. A fourth-order calculation requires five numerical Hessian matrices, each calculated using a different step size. Calculations were then performed at four displacements along each of the normal mode eigenvectors, $\Delta q= \pm 0.8$ and $\Delta q= \pm 1.6$. At each of the $4 F$ displaced geometries, 3 Hessian matrices were calculated numerically in order to apply second-order Richardson extrapolation to the Hessian matrix elements. 0.01a was the smallest step size used in these calculations. First-order Richardson extrapolation was then applied using these converged Hessian elements at both $\Delta q$ displacements to calculate third and fourth potential derivatives. This calculation will be denoted "Q1 H2," since Hessian elements at each of the displaced geometries were calculated at second order and derivatives along the normal mode coordinates $Q$ were calculated at first order. This is the most computationally expensive method applied in this study, requiring 149 TS Hessian matrix calculations. At this level of accuracy, increasing the size of the system requires 36 additional Hessian matrix calculations per additional atom.

The rate constants calculated using this set of anharmonic constants are presented in Fig. 3 and Table IV. Rate constants calculated previously using a QRS method, MultiConfigurational Time-Dependent Hartree (MCTDH), on two different potential energy surfaces $^{38,40}$ and using RPMD on another PES ${ }^{59}$ are also presented in Fig. 3 for comparison. Results from SCTST are somewhat greater than those from other studies, differing from RPMD results by a factor of 3.08 and from MCDTH results by factors of 2.75 and 6.23 at $300 \mathrm{~K}$. This level of agreement is comparable to that among results from the other studies.

In order to evaluate the extent to which Richardson extrapolation affects rate constants calculated using SCTST, another calculation was performed without Richardson extrapolation. Hessians were calculated at displacements of $\Delta q= \pm 0.8$ along different eigenvectors than in the "Q1 H2" calculation, as the Hessian calculated at the TS without Richardson extrapolation differs from the fourth-order Hessian. It was impossible to calculate a density of states for the bound vibrational modes of the TS using the resulting set of anharmonic constants because the first derivatives of the energy in the bound modes $E_{\{n\}^{\prime}}$ with respect to each of the $F-1$ quantum numbers were all negative in the zero-point configuration. These derivatives must be non-negative for all configurations $\{n\}^{\prime}$ considered in the calculation of the CRP, as this ensures that the quantum number of each mode does not exceed its dissociation limit, as discussed in detail by Nguyen and Barker. ${ }^{110}$ The fact that the zero- 
ABLE IV. Rate constants for reaction (1), in units $\mathrm{cm}^{3}$ molecule $\mathrm{s}^{-1} \mathrm{~s}^{-1}$, calculated by TST and $\mathrm{SCTST}^{a}$

\begin{tabular}{|c|c|c|c|c|c|}
\hline$T(\mathrm{~K})$ & TST & $\begin{array}{l}\text { SCTST, } \\
\text { Q1 H2 }\end{array}$ & $\begin{array}{l}\text { SCTST, } \\
\text { Q1 H0 }\end{array}$ & $\begin{array}{c}\text { 1-D SCTST, } \\
\text { Hessians }\end{array}$ & $\begin{array}{c}\text { 1-D SCTST, } \\
\text { energies }\end{array}$ \\
\hline 200 & $1.30(-25)$ & $5.33(-21)$ & $3.94(-21)$ & & $3.96(-22)$ \\
\hline 250 & $1.15(-22)$ & $5.54(-20)$ & $4.78(-20)$ & & $1.13(-20)$ \\
\hline 300 & $1.08(-20)$ & $5.23(-19)$ & $5.12(-19)$ & & $2.14(-19)$ \\
\hline 350 & $2.81(-19)$ & $4.01(-18)$ & $4.24(-18)$ & & $2.41(-18)$ \\
\hline 400 & $3.31(-18)$ & $2.33(-17)$ & $2.58(-17)$ & 17) & $1.72(-17)$ \\
\hline 450 & $2.30(-17)$ & $1.04(-16)$ & 1.18 & -17) & $8.56(-17)$ \\
\hline 500 & $1.10(-16)$ & $3.70(-16)$ & & $3.24(-16)$ & $3.25(-16)$ \\
\hline 600 & $1.20(-15)$ & $2.78(-15)$ & & $2.61(-15)$ & $2.63(-15)$ \\
\hline 700 & $6.93(-15)$ & $1.28(-14)$ & & $1.25(-14)$ & $1.26(-14)$ \\
\hline 800 & $2.67(-14)$ & $4.25(-14)$ & $3(-14)$ & $4.27(-14)$ & $4.31(-14)$ \\
\hline 1000 & $1.89(-13)$ & & $4(-13)$ & $2.61(-13)$ & $2.63(-13)$ \\
\hline $\mathrm{CH}_{4}$ & 1 & & 19 & 1 & 1 \\
\hline $\mathrm{TS}$ & 1 & 149 & 53 & 17 & 5 \\
\hline
\end{tabular}

point configuration was determined to be beyond the dissociation limits for all modes implies that the anharmonic constants used in this calculation were not realistic. Rate constants therefore could not be calculated in this case.

As discussed previously, the number of Hessian matrix calculations required to perform a calculation at the "Q1 H2" level of accuracy increases significantly with system size. Furthermore, the cost of each individual numerical Hessian matrix calculation increases with system size. It is therefore desirable to find a method at an intermediate level of accuracy that scales more favorably but yields accurate results, as determined by agreement with results from the "Q1 H2" calculation. We therefore performed further calculations and found that results from a "Q1 H0" calculation with the same step sizes exhibited the best agreement. This treatment only differs from the "Q1 H2" scheme in that only one numer- 


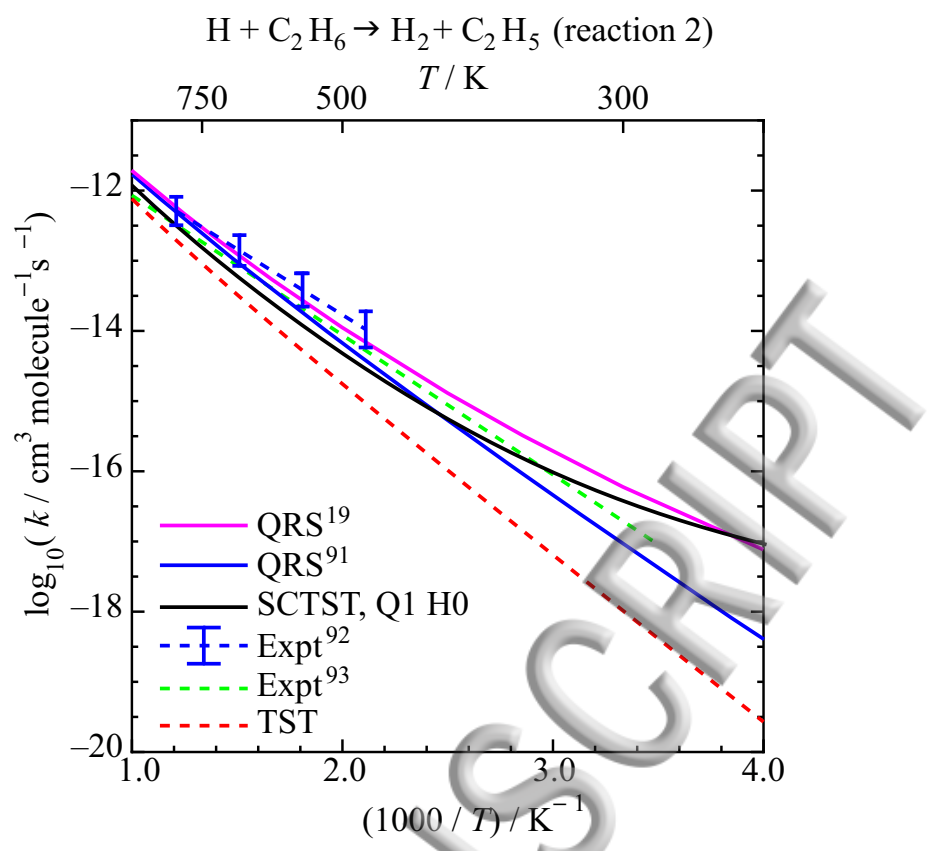

FIG. 4. Rate constants for reaction (2) calculated in the "Q1 H0" scheme described in the text (black curve) shown in comparison with results from previous 2-D QRS studies ${ }^{19,91}$ (magenta and blue curves), and other experimental studies ${ }^{92,93}$ (dashed green and blue curves). Results calculated in this study using conventional TST are included as well (red dashed curve).

ical Hessian matrix calculation was performed at each displaced geometry. Rate constants calculated with this set of TS anharmonic constants can be found in Fig. 3 and Table IV. These results differ from the "Q1 H2" results by $14 \%$ at $250 \mathrm{~K}$.

The number of ab initio Hessian matrix calculations required for each of the rate constant calculations discussed in this section are listed in Table IV in order to give a sense of their relative computational costs. Numerical Hessian matrices calculated at different step sizes and used to calculate higher-order matrix elements by Richardson extrapolation are considered separate Hessian calculations. The "Q1 H0" calculation requires 36\% of the Hessian calculations required for the "Q1 H2" calculation, and yet the resulting rate constants are comparable in accuracy. This reduction in the number of Hessian calculations required may yield substantial efficiency gains for larger systems with more DOFs. 


\section{$\mathrm{H}+\mathrm{C}_{2} \mathrm{H}_{6} \longrightarrow \mathrm{H}_{2}+\mathrm{C}_{2} \mathrm{H}_{5}$ (reaction 2)}

Rate constants for reaction (2) calculated in the "Q1 H0" scheme described above are presented in Fig. 4 and Table V. In these calculations, the DOFs corresponding to internal rotation about the $\mathrm{C}-\mathrm{C}$ bond (torsional modes) in both the TS and $\mathrm{C}_{2} \mathrm{H}_{6}$ were not included in anharmonic constant calculations and were instead treated using hindered rotor partition functions. These results exhibit reasonable agreement with those from a previous 2-D QRS calculation by von Horsten et al., ${ }^{19}$ differing by $19 \%$ at $250 \mathrm{~K}$ but by more at higher temperatures (factor of 2.40 at $500 \mathrm{~K}$ ). Results from an earlier 2-D QRS calculation ${ }^{91}$ exhibit better agreement at higher temperatures but are 16.2 times less than the SCTST rate constants from this study at $260 \mathrm{~K}$. Results from two experimental studies, ${ }^{92,93}$ as well as from conventional TST, are included for comparison. In TST calculations, torsional modes were treated as in SCTST calculations.

\section{One-Dimensional SCTST Calculations}

A 1-D SCTST calculation depends only on the third and fourth derivatives of the PES along the reaction mode, which are used to calculate the anharmonic constant for the reaction mode, $x_{F F}$. 1-D SCTST calculations were performed using the same derivatives that were used in the "Q1 H2"/ and "Q1 H0" FD calculations for reactions (1) and (2), respectively. The resulting rate constants are labeled as "1-D SCTST, Hessians" in Fig. 5 and Tables IV and V, as they were calculated by differentiating Hessian matrix elements. For both reactions (1) and (2), results from 1-D calculations agree sufficiently well with those from FD calculations. This 1-D method underestimates rate constants by factors of 4.54 and 2.14 at $250 \mathrm{~K}$, respectively.

These discrepancies can be understood by considering the potential barriers associated with each of these calculations, which are presented in Figs. 5b and 5d. Only the potential barriers corresponding to the ground-state configuration of $\{n\}^{\prime}$ are shown for the FD calculations. The ground-state configuration is the only configuration sampled in the lowest-energy bin of the density of states (Eq. 36), as is required in the Wang-Landau algorithm. ${ }^{110}$ This configuration contributes most significantly to calculated rate constants due to the presence of a Boltzmann factor in Eq. (28). 


$$
\mathrm{H}+\mathrm{CH}_{4} \rightarrow \mathrm{H}_{2}+\mathrm{CH}_{3} \text { (reaction 1) }
$$

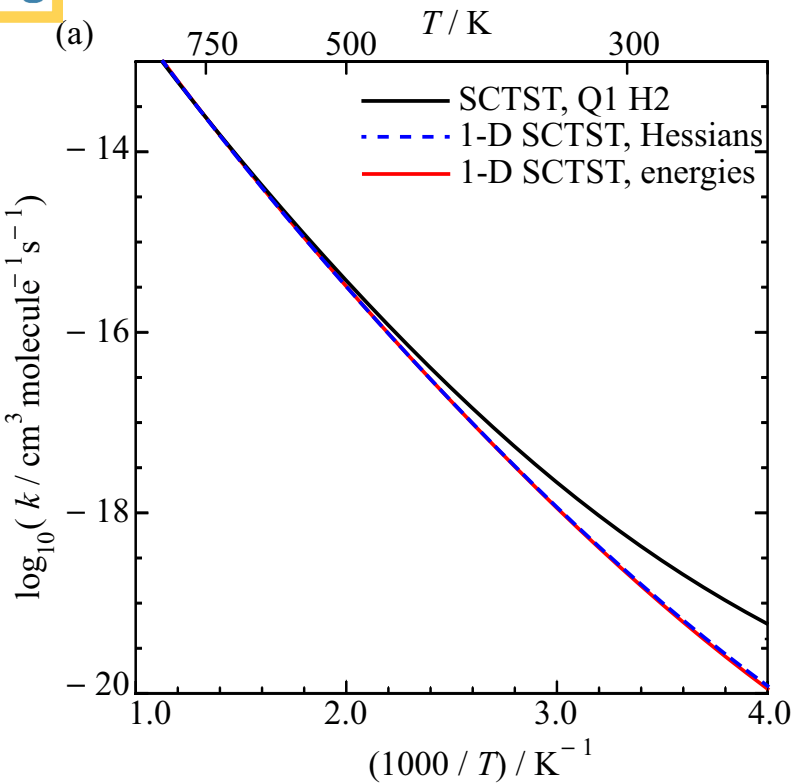

(b)

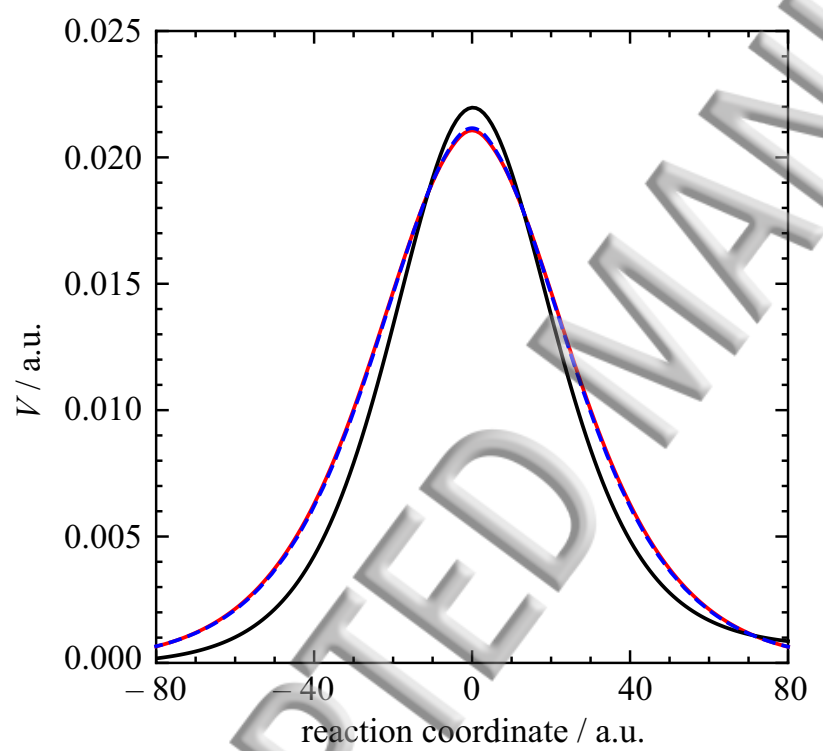

$\mathrm{H}+\mathrm{C}_{2} \mathrm{H}_{6} \rightarrow \mathrm{H}_{2}+\mathrm{C}_{2} \mathrm{H}_{5}$ (reaction 2)

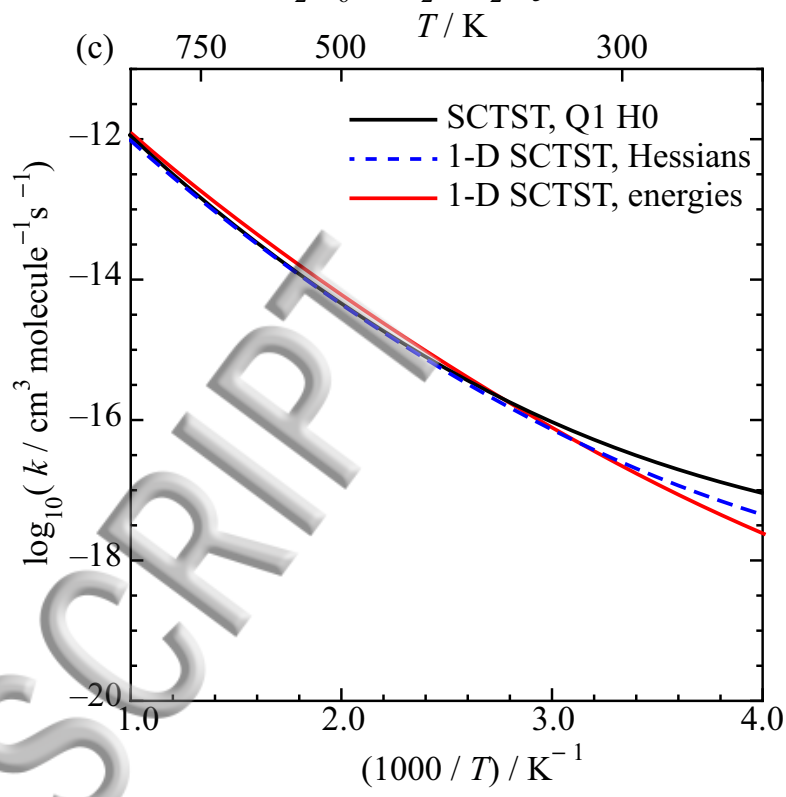

(d)

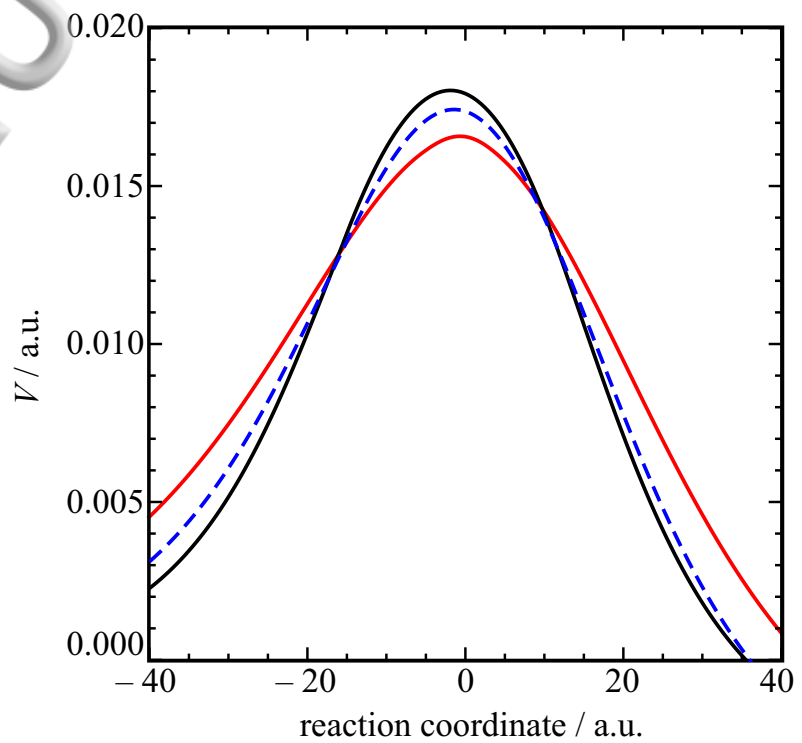

FIG. 5. (a) Rate constants for reaction (1) calculated using FD and 1-D SCTST. The results labeled "1-D SCTST, Hessians" were calculated using anharmonic constants obtained from Hessian matrices at displaced geometries (dashed blue curve). Those labeled "1-D SCTST, energies" were calculated using single-point energy calculations along the reaction mode (red curve). (b) The corresponding vibrationally adiabatic potential barriers used to calculate these rate constants. The FD barrier is presented for the case in which the quantum numbers of all bound modes are 0 . (c) \& (d) The same results for reaction (2). 
ABLE V. Rate constants for reaction (2), in units $\mathrm{cm}^{3}$ molecule ${ }^{-1} \mathrm{~s}^{-1}$, calculated using the methods discussed in this study. ${ }^{a}$

\begin{tabular}{ccccc}
\hline \hline & & SCTST, & 1-D SCTST & 1-D SCTST \\
$T(\mathrm{~K})$ & TST & Q1 H0 & (Hessians $)$ & (energies $)$ \\
\hline 200 & $8.36(-23)$ & $2.50(-18)$ & $8.30(-19)$ & $2.08(-19)$ \\
250 & $2.09(-20)$ & $9.51(-18)$ & $4.45(-18)$ & $2.37(-18)$ \\
300 & $8.47(-19)$ & $3.88(-17)$ & $2.47(-17)$ & $2.14(-17)$ \\
350 & $1.22(-17)$ & $1.56(-16)$ & $1.21(-16)$ & $1.34(-16)$ \\
400 & $9.16(-17)$ & $5.66(-16)$ & $4.86(-16)$ & $6.03(-16)$ \\
450 & $4.48(-16)$ & $1.78(-15)$ & $1.60(-15)$ & $2.08(-15)$ \\
500 & $1.62(-15)$ & $4.85(-15)$ & $4.43(-15)$ & $5.88(-15)$ \\
600 & $1.16(-14)$ & $2.50(-14)$ & $2.27(-14)$ & $3.03(-14)$ \\
700 & $4.93(-14)$ & $9.00(-14)$ & $7.97(-14)$ & $1.04(-13)$ \\
800 & $1.51(-13)$ & $2.51(-13)$ & $2.15(-13)$ & $2.77(-13)$ \\
1000 & $7.70(-13)$ & $1.19(-12)$ & $9.53(-13)$ & $1.18(-12)$ \\
\hline \hline TS & Number of ab initio Hessian calculations required & 1 \\
\hline \hline
\end{tabular}

The FD barriers for reactions (1) and (2) are higher than the respective 1-D barriers but are also narrower, which gives rise to greater reaction probabilities at low energies in FD calculations, and therefore greater rate constants, relative to the "1-D, Hessians" calculations. The width of each barrier is determined by the effective reaction mode frequency $\Omega$ and reaction mode anharmonic constant $x_{F F}$ used to construct it. Both of these parameters incorporate coupling between the reaction mode and bound modes through cross-derivatives of the PES. Therefore, this coupling can be thought to narrow the FD barrier relative to the 1-D barrier. Differences in the heights of these barriers arise from differences between the harmonic and anharmonic ZPEs of the reactants and TS.

Performing a 1-D calculation in this way requires two numerical Hessian matrix calculations at displaced geometries. The number of single-point energy calculations required 
Publishifog these calculations generally increases with system size. In principle, however, only four single-point energy calculations along the reaction mode are required to calculate the third and fourth PES derivatives required for a 1-D SCTST calculation, in addition to one at the TS. An additional calculation was performed in this way, using single-point energies calculated at the MP2/cc-pVTZ level of theory with a step size of $0.02 \mathrm{Da}^{1 / 2} a_{0}$, which was found to yield the best agreement with FD rate constants. Second-order Richardson extrapolation was found not to significantly change these results. Rate constants calculated from the resulting anharmonic constants are presented in Fig. 5 and Tables IV and V and labeled as "1-D SCTST, energies." These results exhibited a high level of agreement with the "1-D SCTST, Hessians" results, which is reflected in the similarity of the corresponding potential barriers (Figs. 5b and 5d).

In the case of reaction (1), calculating the FD "Q1 H2" rate constants required 144 Hessian matrix calculations at displaced geometries, which in total required 33,504 singlepoint energy calculations, more than 8000 times more than the 4 required for the " $1-\mathrm{D}$ SCTST, energies" calculation. For reaction (2), this difference in the computational costs of the FD and 1-D calculations beyond those required for a conventional TST calculation differed by a factor of approximately 10,000 .

\section{CONCLUSIONS}

This study inyestigates the accuracy of a 1-D SCTST approach to approximating the rate constants of chemical reactions. Rate constants from 1-D SCTST calculations are compared to those from full-dimensional (FD) calculations, which require third and fourth derivatives of the PES. A practical procedure for evaluating these derivatives numerically was discussed, and a method (Richardson extrapolation) for systematically improving their accuracy was presented and applied. Third and fourth derivatives along only the reaction mode are required for 1-D SCTST calculations, so these were also obtained by differentiating single-point energies.

FD SCTST rate constants calculated for both reactions agreed well with results from previous theoretical and experimental studies. FD calculations for the $\mathrm{H}+\mathrm{CH}_{4}$ reaction were performed at three different levels of accuracy, as specified by the order of Richardson extrapolation applied to different derivatives. Calculations at the lowest level of accuracy, 
Publishiwgich involved the least computational expense, failed because the anharmonic constants calculated by this method were determined not to be physically realistic. Calculations at an intermediate level of accuracy agreed very well with those at the highest level of accuracy but required $64 \%$ fewer Hessian matrix calculations. Rate constant calculations for the $\mathrm{H}+\mathrm{C}_{2} \mathrm{H}_{6}$ reaction were therefore performed at this intermediate level of accuracy. These results suggest the applicability of Richardson extrapolation to SCTST calculations as an alternative to existing methods that depend upon analytical derivatives.

For both reactions, the two 1-D SCTST methods yielded rate constants in acceptable agreement with each other and with FD rate constants. The 1-D method that used singlepoint energy calculations was significantly more efficient than the FD methods implemented in this study, as it requires at minimum 4 single-point energy calculations in addition to those required for a conventional TST calculation. If necessary, this approach can be systematically improved by Richardson extrapolation. The low computational expense of this 1-D method suggests its applicability as a method for approximating the rate constants for systems significantly larger than those that could previously be treated by quantum scattering approaches.

\section{ACKNOWLEDGMENTS}

X. Shan and D. C. Clary acknowledge financial support from the Leverhulme Trust (Project Grant No. RPG-2013-321). S. Greene acknowledges financial support from the Rhodes Trust through a Rhodes Scholarship for graduate study. We thank Professor Todd Martinez for useful discussions on Richardson extrapolation.

\section{REFERENCES}

1M. Prather and A. H. Jaffe, J. Geophys. Res.: Atmos. 95, 3473 (1990).

${ }^{2}$ A. R. Douglass, M. R. Schoeberl, R. S. Stolarski, J. W. Waters, J. M. Russell, A. E. Roche, and S. T. Massie, J. Geophys. Res.: Atmos. 100, 13967 (1995).

${ }^{3}$ H. A. Michelsen, C. R. Webster, G. L. Manney, D. C. Scott, J. J. Margitan, R. D. May, F. W. Irion, M. R. Gunson, J. M. Russell, and C. M. Spivakovsky, J. Geophys. Res.: Atmos. 104, 26419 (1999). 
Publishinģ I. L. Santee, L. Froidevaux, G. L. Manney, W. G. Read, J. W. Waters, M. P. Chipperfield,

A. E. Roche, J. B. Kumer, J. L. Mergenthaler, and J. M. Russell, J. Geophys. Res. 101, 18835 (1996).

${ }^{5}$ J. Gao and D. G. Truhlar, Annu. Rev. Phys. Chem. 53, 467 (2002).

${ }^{6}$ R. M. Navarro Yerga, M. C. Álvarez Galván, F. del Valle, J. Villoria de la Mano, and J. L. G. Fierro, ChemSusChem 2, 471 (2009).

${ }^{7}$ A. J. Leenheer and H. A. Atwater, J. Electrochem. Soc. 157, B1290 (2010).

${ }^{8}$ T. Hisatomi, J. Kubota, and K. Domen, Chem. Soc. Bev, 43, 7520 (2014).

${ }^{9}$ S. C. Althorpe and D. C. Clary, Annu. Rev. Phys. Chem. 54, 493 (2003).

${ }^{10}$ N. Balakrishnan, C. Kalyanaraman, and N. Sathyamurthy, Phys. Rep. 280, 79 (1997).

${ }^{11}$ H. Tal-Ezer and R. Kosloff, J. Chem. Phys. 81, 3967 (1984).

${ }^{12}$ M. D. Feit and J. A. Fleck, J. Chem. Phys. 80, 2578 (1984).

${ }^{13}$ W. Hu and G. C. Schatz, J. Chem. Phys. 125, 132301 (2006).

${ }^{14}$ G. Nyman and H.-G. Yu, Rep. Prog Phys. 63, 1001 (2000).

${ }^{15}$ W. H. Miller, Annu. Rev. Phys. Chem. 41, 245 (1990).

${ }^{16}$ J. M. Bowman, J. Phys. Chem. 95, 4960 (1991).

${ }^{17}$ J. M. Bowman, Theor. Chem. Acc. 108, 125 (2002).

${ }^{18}$ S. T. Banks, C. S. Tautermann, S. M. Remmert, and D. C. Clary, J. Chem. Phys. 131, 044111 (2009).

${ }^{19}$ H. F. von Horsten/S. T. Banks, and D. C. Clary, J. Chem. Phys. 135, 094311 (2011).

${ }^{20}$ B. Lasorne, M. A. Robb, and G. A. Worth, Phys. Chem. Chem. Phys. 9, 3210 (2007).

${ }^{21}$ G. A. Worth, H.-D. Meyer, H. Köppel, L. S. Cederbaum, and I. Burghardt, Int. Rev. Phys. Chem. 27, 569 (2008).

${ }^{22}$ H.-D. Meyer, F. Gatti, and G. Worth, eds., Multidimensional Quantum Dynamics: MCTDH Theory and Applications (John Wiley \& Sons, Weinheim, 2009).

${ }^{23}$ T. J. Frankcombe, M. A. Collins, and G. A. Worth, Chem. Phys. Lett. 489, 242 (2010).

${ }^{24}$ J. M. Bowman, B. J. Braams, S. Carter, C. Chen, G. Czakó, B. Fu, X. Huang, E. Kamarehik, A. R. Sharma, B. C. Shepler, Y. Wang, and Z. Xie, J. Phys. Chem. Lett. 1, 1866 (2010).

${ }^{25}$ J. M. Bowman, G. Czako, and B. Fu, Phys. Chem. Chem. Phys. 13, 8094 (2011).

${ }^{26}$ W. Koch and T. J. Frankcombe, Phys. Rev. Lett. 110, 263202 (2013).

${ }^{27}$ W. Koch and D. H. Zhang, J. Chem. Phys. 141, 021101 (2014). 
Publishirigr . Conte, P. L. Houston, and J. M. Bowman, J. Chem. Phys. 140, 151101 (2014).

${ }^{29}$ R. Welsch and U. Manthe, J. Chem. Phys. 141, 174313 (2014).

${ }^{30}$ U. Manthe, J. Chem. Phys. 142, 244109 (2015).

${ }^{31}$ F. Huarte-Larrañaga and U. Manthe, J. Chem. Phys. 113, 5115 (2000).

${ }^{32}$ F. Huarte-Larrañaga and U. Manthe, J. Phys. Chem. A 105, 2522 (2001).

${ }^{33}$ T. Wu, H.-J. Werner, and U. Manthe, Science 306, 2227 (2004).

${ }^{34}$ T. Wu, H.-J. Werner, and U. Manthe, J. Chem. Phys. 124, 164307 (2006).

${ }^{35}$ G. Schiffel and U. Manthe, J. Chem. Phys. 132, 084103 (2010).

${ }^{36}$ R. van Harrevelt, G. Nyman, and U. Manthe, J. Chem. Phys. 126, 084303 (2007).

${ }^{37}$ R. Welsch and U. Manthe, J. Chem. Phys. 141, 051102 (2014).

${ }^{38}$ R. Welsch and U. Manthe, J. Chem. Phys. 137, 244106 (2012).

${ }^{39}$ R. Welsch and U. Manthe, J. Chem. Phys. 138, 164118 (2013).

${ }^{40}$ R. Welsch and U. Manthe, J. Chem. Phys. 142, 064309 (2015).

${ }^{41}$ D. G. Truhlar, A. D. Isaacson, and B. C. Garrett, in Theory of Chemical Reaction Dynamics, Vol. IV, edited by M. Baer (CRC Press, Boca Raton, 1985) Chap. 2, pp. 65-137.

${ }^{42}$ B. C. Garrett and D. G. Truhlar, in Theory and Applications of Computational Chemistry: The First Forty Years, edited by C. E. Dykstra, G. Frenking, K. S. Kim, and G. E. Scuseria (Elsevier, Amsterdam, 2005) Chap. 5, pp. 67-87.

${ }^{43}$ A. Fernandez-Ramos, B. A. Ellingson, B. C. Garrett, and D. G. Truhlar, in Reviews in Computational Chemistry, Vol. 23, edited by K. B. Lipkowitz and T. R. Cundari (John Wiley \& Sons, Inc. Hoboken, 2007) Chap. 3, pp. 125-232.

${ }^{44}$ D. J. Arseneau, D. G. Fleming, Y. Li, J. Li, Y. V. Suleimanov, and H. Guo, J. Phys. Chem. B 120, 1641 (2016).

${ }^{45}$ Y. N.Suleimanov and J. Espinosa-Garcia, J. Phys. Chem. B 120, 1418 (2016).

${ }^{46}$ M. Ng, D. K. Mok, E. P. Lee, and J. M. Dyke, Mol. Phys. 113, 1511 (2015).

${ }^{47}$ S. Gong, Q. Luo, and G. M. Wei, J. Phys. Chem. A 119, 4746 (2015).

${ }^{48}$ E. Gonzalez-Lavado, J. C. Corchado, Y. V. Suleimanov, W. H. Green, and J. EspinosaGarcia, J. Phys. Chem. A 118, 3243 (2014).

${ }^{49}$ R. Meana-Pañeda, D. G. Truhlar, and A. Fernández-Ramos, J. Chem. Theory Comput. 6, 3015 (2010).

${ }^{50}$ R. Meana-Pañeda, D. G. Truhlar, and A. Fernández-Ramos, J. Chem. Theory Comput. 
${ }^{51}$ T. V. Albu, J. C. Corchado, and D. G. Truhlar, J. Phys. Chem. A 105, 8465 (2001).

${ }^{52}$ T. C. Allison and D. G. Truhlar, in Modern Methods for Multidimensional Dynamics Computations in Chemistry, edited by D. L. Thompson (World Scientific, Singapore, 1998) pp. 618-712.

${ }^{53}$ S. Jang and G. A. Voth, J. Chem. Phys. 112, 8747 (2000).

${ }^{54}$ E. Geva, Q. Shi, and G. A. Voth, J. Chem. Phys. 115, 9209 (2001).

${ }^{55}$ I. Navrotskaya, Q. Shi, and E. Geva, Isr. J. Chem. 42, 225 (2002).

${ }^{56}$ G. A. Voth, D. Chandler, and W. H. Miller, J. Chem. Phys. 91, 7749 (1989).

${ }^{57}$ I. R. Craig and D. E. Manolopoulos, J. Chem. Phys. 122, 084106 (2005).

${ }^{58}$ I. R. Craig and D. E. Manolopoulos, J. Chem(Phys. 123, 034102 (2005).

${ }^{59}$ Q. Meng, J. Chen, and D. H. Zhang, J. Chem. Phys 143, 101102 (2015).

${ }^{60}$ Y. V. Suleimanov, R. Collepardo-Guevara, and D. E. Manolopoulos, J. Chem. Phys. 134, 044131 (2011).

${ }^{61}$ W. H. Miller, J. Chem. Phys. 62, 1899 (1975).

${ }^{62}$ W. H. Miller, Faraday Discuss, Chem.Soe. 62, 40 (1977).

${ }^{63}$ W. H. Miller, R. Hernandez, N. C. Handy, D. Jayatilaka, and A. Willetts, Chem. Phys. Lett. 172, 62 (1990).

${ }^{64}$ M. J. Cohen, N. C. Handy, R. Hernandez, and W. H. Miller, Chem. Phys. Lett. 192, 407 (1992).

${ }^{65}$ M. J. Cohen, A. Willetts, and N. C. Handy, Chem. Phys. Lett. 223, 459 (1994).

${ }^{66}$ T. L. Nguyen, J. F. Stanton, and J. R. Barker, J. Phys. Chem. A 115, 5118 (2011).

${ }^{67}$ J. R. Barker, D. L. Nguyen, and J. F. Stanton, J. Phys. Chem. A 116, 6408 (2012).

${ }^{68}$ S. M. Greene, X. Shan, and D. C. Clary, J. Phys. Chem. A 119, 12015 (2015).

${ }^{69}$ S. M. Greene, X. Shan, and D. C. Clary, J. Chem. Phys. 144, 084113 (2016).

${ }^{70}$ A. F Wagner, J. Phys. Chem. A 117, 13089 (2013).

${ }^{71}$ W. Schneider and W. Thiel, Chem. Phys. Lett. 157, 367 (1989).

${ }^{72}$ J. Neugebauer and B. A. Hess, J. Chem. Phys. 118, 7215 (2003).

${ }^{73}$ V. Barone, J. Chem. Phys. 122, 14108 (2005).

${ }^{74}$ R. D. Amos, N. C. Handy, W. H. Green, D. Jayatilaka, A. Willetts, and P. Palmieri, J. Chem. Phys. 95, 8323 (1991).

${ }^{75}$ A. L. L. East, W. D. Allen, and S. J. Klippenstein, J. Chem. Phys. 102, 8506 (1995). 
Publishinģfr. Burcl, N. C. Handy, and S. Carter, Spectrochim. Acta, Part A 59, 1881 (2003).

${ }^{77}$ H.-J. et al.. Werner, "MOLPRO, a package of ab initio programs, version 2010.2," .

${ }^{78}$ Y. Shao, Z. Gan, E. Epifanovsky, A. T. B. Gilbert, M. Wormit, J. Kussmann, A. W. Lange, A. Behn, J. Deng, X. Feng, D. Ghosh, M. Goldey, P. R. Horn, L. D. Jacobson, I. Kaliman, R. Z. Khaliullin, T. Kús, A. Landau, J. Liu, E. I. Proynov, Y. M. Rhee, R. M. Richard, M. A. Rohrdanz, R. P. Steele, E. J. Sundstrom, H. L. Woodcock III, P. M. Zimmerman, D. Zuev, B. Albrecht, E. Alguire, B. Austin, G. J. O. Beran, Y. A. Bernard, E. Berquist, K. Brandhorst, K. B. Bravaya, S. T. Brown, D. Casanova, C.-M. Chang, Y. Chen, S. H. Chien, K. D. Closser, D. L. Crittenden, M. Diedenhofen, R. A. DiStasio Jr., H. Dop, A. D. Dutoi, R. G. Edgar, S. Fatehi, L. Fusti-Molnar, A. Ghysels, A. Golubeva-Zadorozhnaya, J. Gomes, M. W. D. Hanson-Heine, P. H. P. Harbach, A. W. Hauser, E. G. Hohenstein, Z. C. Holden, T-C. Jagau, H. Ji, B. Kaduk, K. Khistyaev, J. Kim, J. Kim, R. A. King, P. Klunzinger, D. Kosenkov, T. Kowalczyk, C. M. Krauter, K. U. Lao, A. Laurent, K. V. Lawler, S. V. Levchenko, C. Y. Lin, F. Liu, E. Livshits, R. C. Lochan, A. Luenser, P. Manohar, S. F. Manzer, S.-P. Mao, N. Mardirossian, A. V. Marenich, S. A. Maurer, N. J. Mayhall, C. M. Oana, R. Olivares-Amaya, D. P. O'Neill, J. A. Parkhill, T. M. Perrine, R. Peverati, P. A. Pieniazek, A. Prociuk, D. R. Rehn, E. Rosta, N. J. Russ, N. Sergueev, S. M. Sharada, S. Sharmaa, D. W. Small, A. Sodt, T. Stein, D. Stück, Y.-C. Su, A. J. W. Thom, T. Tsuchimochi, L. Vogt, O. Vydrov, T. Wang, M. A. Wátson, J. Wenzel, A. White, C. F. Williams, V. Vanovschi, S. Yeganeh, S. R. Yost, Z.-Q. You, I. Y. Zhang, X. Zhang, Y. Zhou, B. R. Brooks, G. K. L. Chan, D. M. Chipman, C. J. Cramer, W. A. Goddard III, M. S. Gordon, W. J. Hehre, A. Klamt, H. F. Schaefer III, M. W. Schmidt, C. D. Sherrill, D. G. Truhlar, A. Warshel, X. Xua, A. Aspuru-Guzik, R. Baer, A. T. Bell, N. A. Besley, J.-D. Chai, A. Dreuw, B. D. Dunietz, T. R. Furlani, S. R. Gwaltney, C.-P. Hsu, Y. Jung, J. Kong, D. S. Lambrecht, W. Liang, C. Ochsenfeld, V. A. Rassolov, L. V. Slipchenko, J. E. Subotnik, T. Van Voorhis, J. M. Herbert, A. I. Krylov, P. M. W. Gill, and M. Head-Gordon, Mol. Phys. 113, 184 (2015). ${ }^{79}$ M. J. Frisch, G. W. Trucks, H. B. Schlegel, G. E. Scuseria, M. A. Robb, J. R. Cheeseman, G. Scalmani, V. Barone, B. Mennucci, G. A. Petersson, H. Nakatsuji, M. Caricato, X. Li, H. P. Hratchian, A. F. Izmaylov, J. Bloino, G. Zheng, J. L. Sonnenberg, M. Hada, M. Ehara, K. Toyota, R. Fukuda, J. Hasegawa, M. Ishida, T. Nakajima, Y. Honda, O. Kitao, H. Nakai, T. Vreven, J. A. Montgomery, Jr., J. E. Peralta, F. Ogliaro, M. Bearpark, 
Publishing]. J. Heyd, E. Brothers, K. N. Kudin, V. N. Staroverov, R. Kobayashi, J. Normand,

K. Raghavachari, A. Rendell, J. C. Burant, S. S. Iyengar, J. Tomasi, M. Cossi, N. Rega, J. M. Millam, M. Klene, J. E. Knox, J. B. Cross, V. Bakken, C. Adamo, J. Jaramillo,

R. Gomperts, R. E. Stratmann, O. Yazyev, A. J. Austin, R. Cammi, C. Pomelli, J. W. Ochterski, R. L. Martin, K. Morokuma, V. G. Zakrzewski, G. A. Voth, P. Salvador, J. J. Dannenberg, S. Dapprich, A. D. Daniels, . Farkas, J. B. Foresman, J. V. Ortiz, J. Cioslowski, and D. J. Fox, "Gaussian 09 Revision E.01," Gaussian, Inc., Wallingford (2009).

${ }^{80}$ M. F. Guest, I. J. Bush, H. J. J. V. Dam, P. Sherwood, J. M. H. Thomas, J. H. V. Lenthe, R. W. A. Havenith, and J. Kendrick, Mol. Phys. 103, 719 (2005).

81 “J. F. Stanton, J. Gauss, M. E. Harding, P. G. Szalay, w.c. f. A. A. Auer, R. J. Bartlett, U. Benedikt, C. Berger, D. E. Bernholdt, Y. J. Bomble, O. Christiansen, M. Heckert, O. Heun, C. Huber, T.-C. Jagan, D. Jonsson, J. Jusélius, K. Klein, W. J. Lauderdale, D. A. Matthews, T. Metzroth, D. P. ONeill, D. R. Price, E. Prochnow, K. Ruud, F. Schiffmann, S. Stopkowicz, J. Vázquez, F. Wang, J. D. Watts and the integral packages MOLECUlE (J. Almlöf, P. R. Taylor); PROPS (P. R. Taylor); ABACUS (T. Helgaker, H. J. Aa. Jensen, P. Jørgensen, J. Olsen); and ECP routines by A. V. Mitin, C. van Wüllen. CFOUR, Coupled-Cluster techniques for Computational Chemistry, a quantum-chemical program package," (2009).

${ }^{82}$ A. Jäckle, M.-C. Heitz, and H.-D. Meyer, J. Chem. Phys. 110, 241 (1999).

${ }^{83}$ G. W. M. Vissers and A. B. McCoy, J. Phys. Chem. A 110, 5978 (2006).

${ }^{84}$ H. B. Schlegel, in Ab Initio Methods in Quantum Chemistry I, Advances in Chemical Physics, Vol. 67, edited by K. Lawley (John Wiley \& Sons, Chichester, 2009) pp. 249286.

${ }^{85}$ P. Pulay, WIREs Comput Mol Sci 4, 169 (2014).

${ }^{86}$ C. Ridders, Adv. Eng. Software 4, 75 (1982).

${ }^{87}$ J. W. Sutherland, M. C. Su, and J. V. Michael, Int. J. Chem. Kinet. 33, 669 (2001).

88 J. Espinosa-García, Phys. Chem. Chem. Phys. 10, 1277 (2008).

${ }^{89}$ M. J. Kurylo, G. A. Hollinden, and R. B. Timmons, J. Chem. Phys. 52, 1773 (1970).

${ }^{90}$ J. Pu and D. G. Truhlar, J. Chem. Phys. 116, 1468 (2002).

${ }^{91}$ B. Kerkeni and D. C. Clary, J. Chem. Phys. 121, 6809 (2004).

${ }^{92}$ M. G. Bryukov, I. R. Slagle, and V. D. Knyazev, J. Phys. Chem. A 105, 6900 (2001). 
${ }^{94}$ D. Jones, P. A. Morgan, and J. H. Purnell, J. Chem. Soc., Faraday Trans. 1 73, 1311 (1977).

${ }^{95}$ T. H. Dunning, J. Chem. Phys. 90, 1007 (1989).

${ }^{96}$ J. R. Barker, Int. J. Chem. Kinet. 33, 232 (2001).

${ }^{97}$ J. R. Barker, N. F. Ortiz, J. M. Preses, L. L. Lohr, A. Maranzana, P. J. Stimac, T. L. Nguyen, and T. J. D. Kumar, "Multiwell-2014.1 software,"(2014), http://aoss .engin . umich.edu/multiwell/.

${ }^{98}$ K. Raghavachari, G. W. Trucks, J. A. Pople, and M. Head-Gordon, Chem. Phys. Lett. 157, 479 (1989).

${ }^{99}$ R. A. Kendall, T. H. Dunning, and R. J. Harrison, J. Chem. Phys. 96, 6796 (1992).

${ }^{100}$ A. M. Mebel, E. W. G. Diau, M. C. Lin, and K. Morokuma, J. Phys. Chem. 100, 7517 (1996), http://dx.doi.org/10.1021/jp953644f.

${ }^{101}$ S. Canneaux, C. Hammaecher, F. Louis, and M. Ribaucour, in Reaction Rate Constant Computations: Theories and Applications, edited by K. Han and T. Chi (Royal Society of Chemistry, Cambridge, 2014) Chap. 2, pp. 34-54.

${ }^{102}$ X. Shan and D. C. Clary, J. Phys. Chem. A 118, 10134 (2014).

${ }^{103}$ L. F. Richardson and J. A. Gaunt, Phil. Trans. R. Soc. Lond. A 226, 299 (1927).

${ }^{104}$ W. H. Press, S. A. Teukolsky, W. T. Vetterling, and B. P. Flannery, Numerical Recipes in C: The Art of Scientific Computing, 2nd ed. (Cambridge University Press, Cambridge, 1992).

${ }^{105}$ I. M. Mills, in Molecular Spectroscopy: Modern Research, edited by C. W. Mathews and K. N. Rao (Academic Press, New York, 1972) Chapter 3.2, pp. 115-140.

${ }^{106}$ W. H. Green, D. Jayatilaka, A. Willetts, R. D. Amos, and N. C. Handy, J. Chem. Phys. 93, $4965(1990)$.

${ }^{107}$ A. Hoy, I. Mills, and G. Strey, Mol. Phys. 24, 1265 (1972).

${ }^{108}$ R. Khairutdinov, K. I. Zamaraev, and V. P. Zhadanov, in Electron Tunneling in Chemistry, Comprehensive Chemical Kinetics, Vol. 30, edited by R. G. Compton (Elsevier, Amsterdam, 1989) pp. 69-110.

${ }^{109}$ J. E. Wollrab, Rotational Spectra and Molecular Structure, Physical Chemistry: a Series of Monographs (Academic Press, Inc., New York, 1967).

${ }^{110}$ T. L. Nguyen and J. R. Barker, J. Phys. Chem. A 114, 3718 (2010). 
Publishiłtff. Wang and D. P. Landau, Phys. Rev. Lett. 86, 2050 (2001).

${ }^{112}$ F. Wang and D. P. Landau, Phys. Rev. E 64, 056101 (2001).

${ }^{113}$ M. Basire, P. Parneix, and F. Calvo, J. Chem. Phys. 129, 081101 (2008).

${ }^{114}$ Y.-Y. Chuang and D. G. Truhlar, J. Chem. Phys. 112, 1221 (2000).

${ }^{115}$ D. J. Griffiths, Introduction to Quantum Mechanics, 2nd ed. (Pearson Education, Inc., Upper Saddle River, 2005).

${ }^{116}$ B. Kerkeni and D. C. Clary, J. Chem. Phys. 120, 2308 (2004). 
a) $\mathrm{TS}$

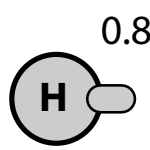

$87382 \AA$

$102.92^{\circ}$

$1.0807 \AA$

b) $\mathrm{CH}_{4}$

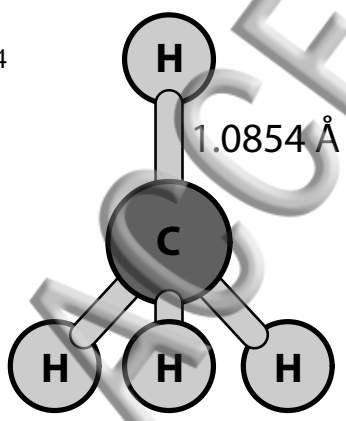

$1.4045 \AA$ H) $C_{3 v}$

c) $\mathrm{CH}_{3}$

d) $\mathrm{H}_{2}$
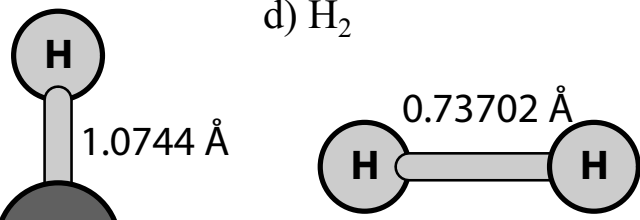
a) $\mathrm{TS}$

(H)

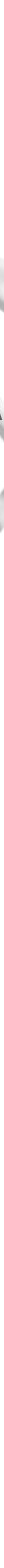

$0.88939 \AA$ 
$\mathrm{H}+\mathrm{CH}_{4} \rightarrow \mathrm{H}_{2}+\mathrm{CH}_{3}$ (reaction 1)

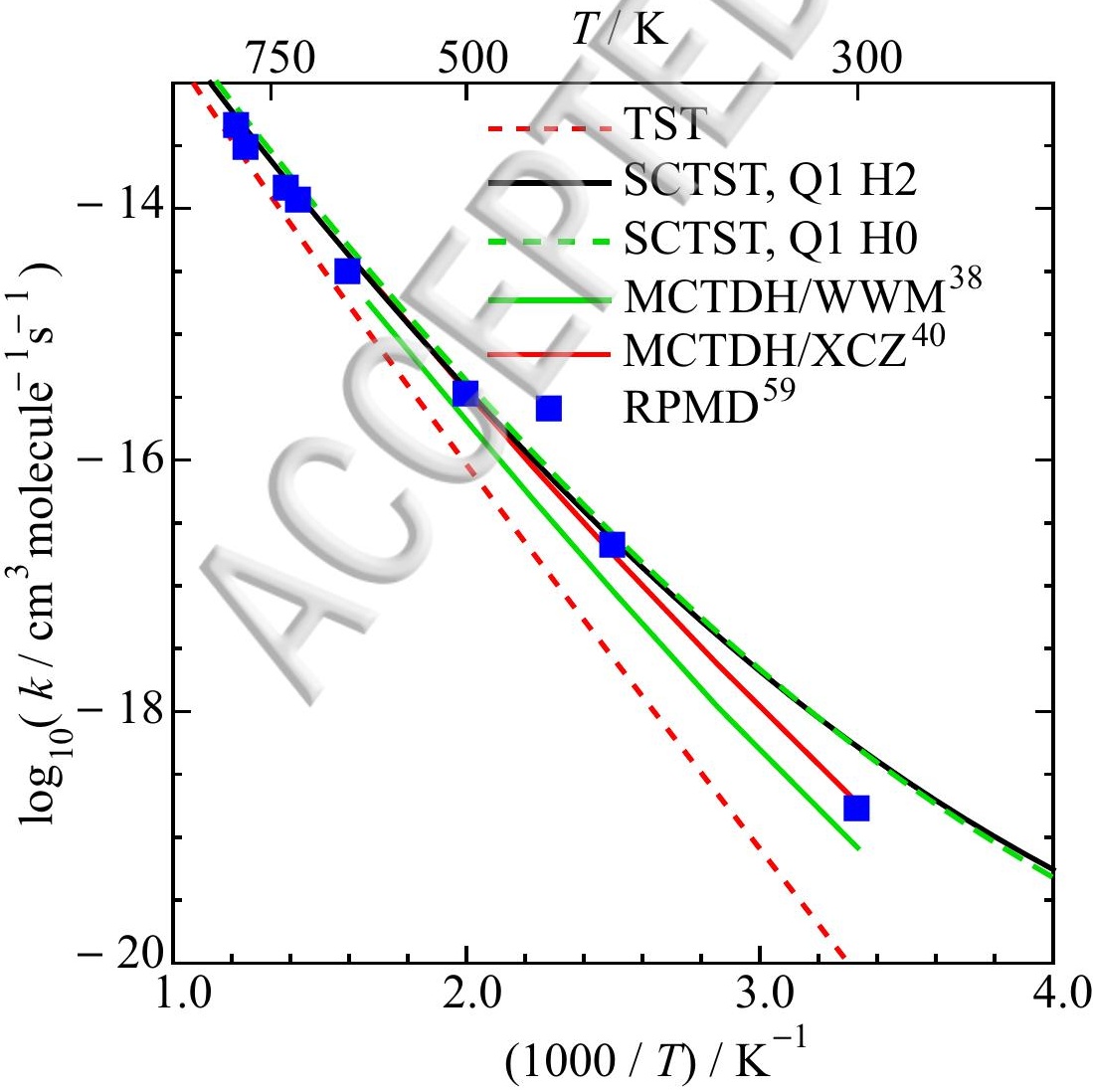


$\mathrm{H}+\mathrm{C}_{2} \mathrm{H}_{6} \rightarrow \mathrm{H}_{2}+\mathrm{C}_{2} \mathrm{H}_{5}$ (reaction 2)

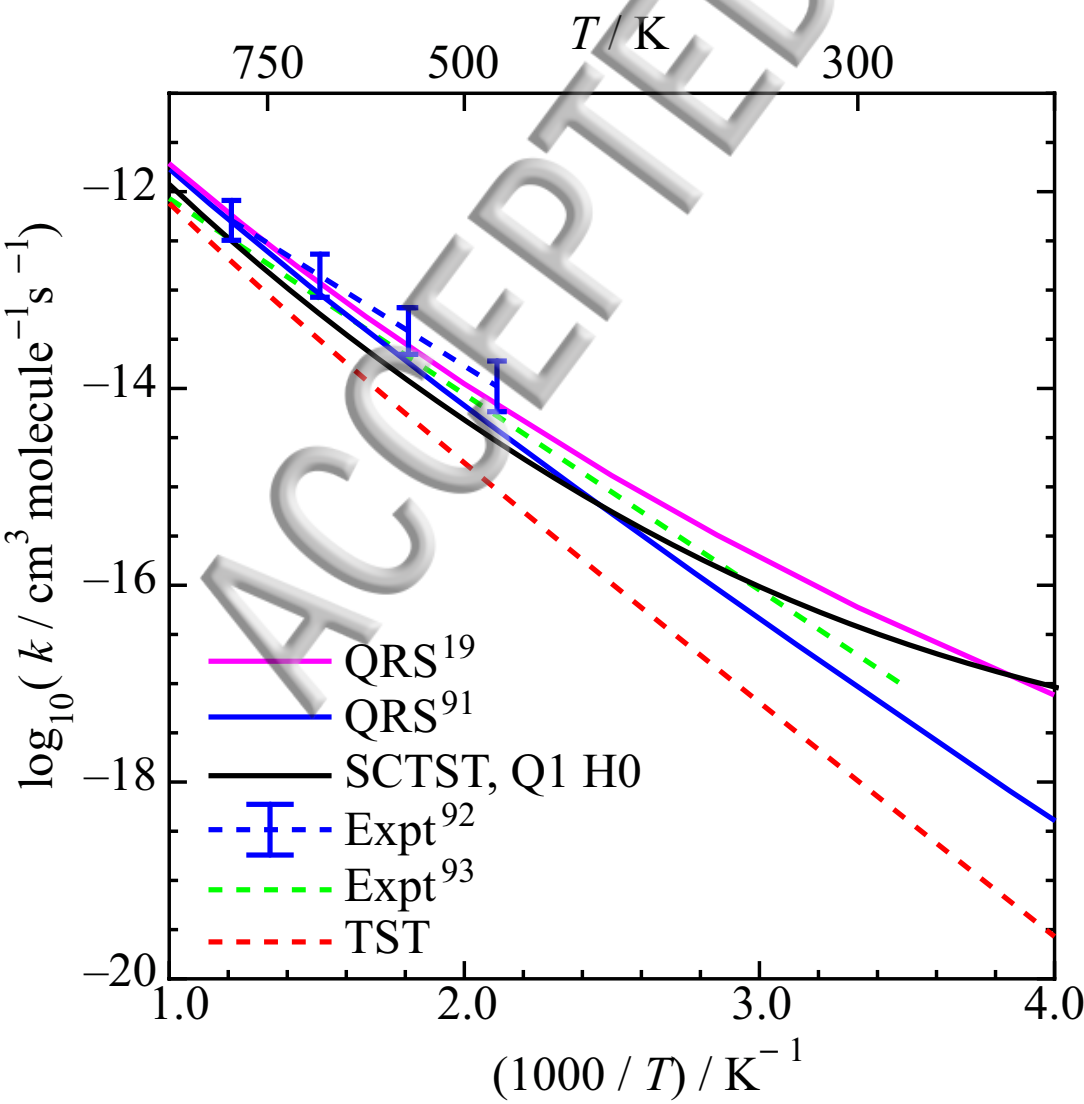


$\mathrm{H}+\mathrm{CH}_{4} \rightarrow \mathrm{H}_{2}+\mathrm{CH}_{3}$ (reaction 1)

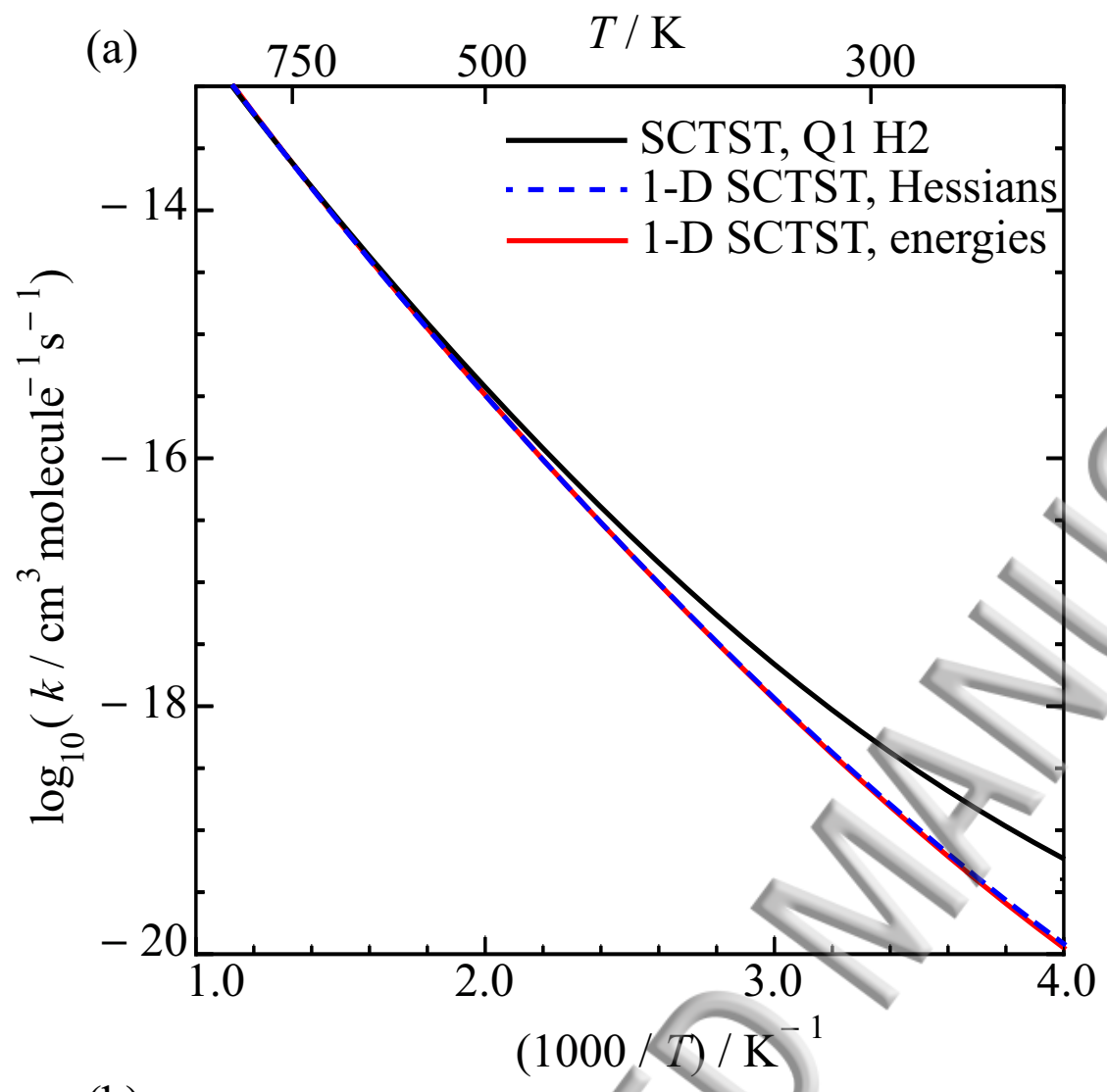

(b)

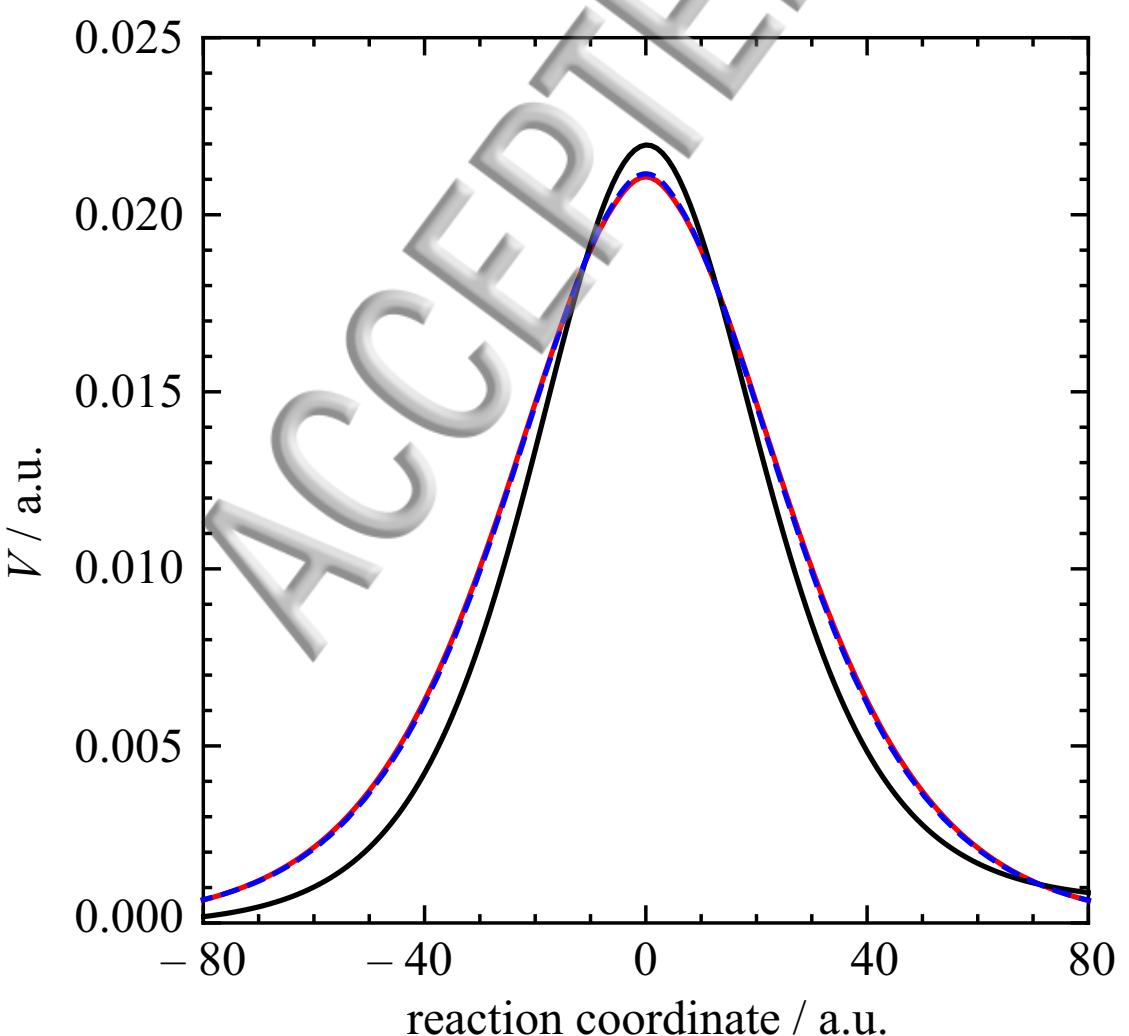

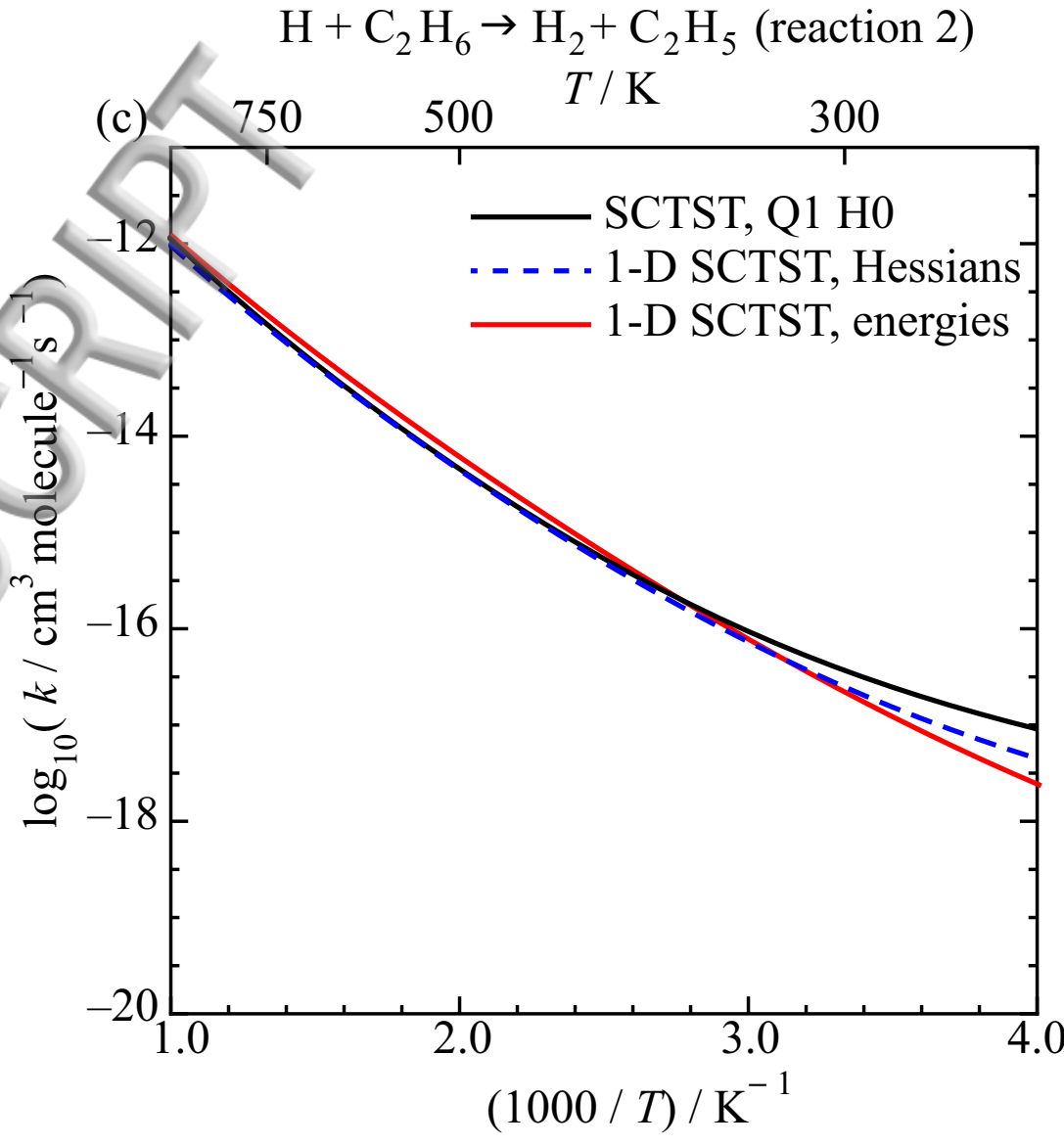

(d)

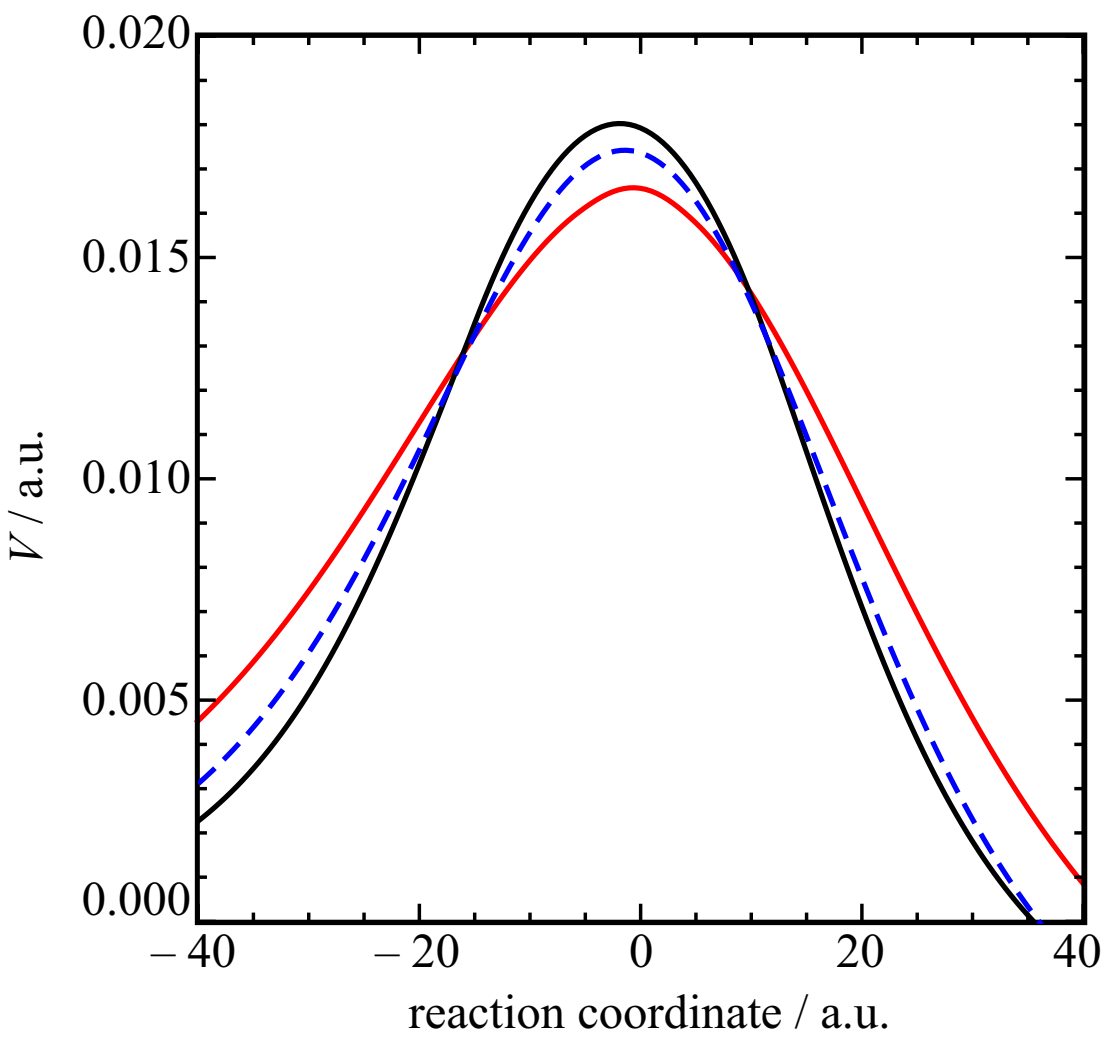

